\title{
Differential Sensorimotor Processing of Vestibulo-Ocular Signals during Rotation and Translation
}

\author{
Dora E. Angelaki, Andrea M. Green, and J. David Dickman \\ Department of Anatomy and Neurobiology, Washington University School of Medicine, St. Louis, Missouri 63110, and \\ Department of Research, Central Institute for the Deaf, St. Louis, Missouri 63110
}

Rotational and translational vestibulo-ocular reflexes (RVOR and TrVOR) function to maintain stable binocular fixation during head movements. Despite similar functional roles, differences in behavioral, neuroanatomical, and sensory afferent properties suggest that the sensorimotor processing may be partially distinct for the RVOR and TrVOR. To investigate the currently poorly understood neural correlates for the TrVOR, the activities of eye movement-sensitive neurons in the rostral vestibular nuclei were examined during pure translation and rotation under both stable gaze and suppression conditions. Two main conclusions were made. First, the $0.5 \mathrm{~Hz}$ firing rates of cells that carry both sensory head movement and motor-like signals during rotation were more strongly related to the oculomotor output than to the vestibular sensory signal during translation. Second, neurons the firing rates of which increased for ipsilaterally versus contralaterally directed eye movements (eye-ipsi

The vestibulo-ocular reflexes (VORs) play an essential role as part of an integrated gaze control system to ensure stable perception of the environment during movement. Despite similar functional goals, however, several lines of evidence suggest that the translational VOR (TrVOR) is likely to be organized differently from the rotational VOR (RVOR). First, the dynamic processing must be different in the two reflexes because over the frequency range of natural head movements, primary otolith afferents encode linear acceleration whereas semicircular canal afferents carry signals most closely related to angular velocity (Fernández and Goldberg, 1971, 1976a-c). Second, the frequency range of effective operation is different for the two reflexes. Although the RVOR elicits compensatory eye movements down to $\sim 0.05 \mathrm{~Hz}$, the TrVOR only exhibits a robust response above $\sim 0.5 \mathrm{~Hz}$ (Paige and Tomko, 1991a; Telford et al., 1997; Angelaki, 1998). Finally, there is increasing evidence that the synaptic organization of the otolith-ocular and canal-ocular pathways is different. Whereas the shortest latency RVOR pathways are mediated dominantly by excitatory projections to the contralateral abducens, the shortest latency TrVOR pathways are instead excitatory to the ipsilateral abducens (Schwindt et al., 1973; Uchino

Received Nov. 16, 2000; revised Feb. 12, 2001; accepted March 7, 2001.

This work was supported by grants from the National Institutes of Health (EY12814, EY10851, and DC04260) and the McDonnell Foundation for higher brain function. We thank Quinn McHenry and Asim Haque for valuable technical assistance, as well as Mimi Galiana and Steve Highstein for reading previous versions of this manuscript.

Correspondence should be addressed to Dr. Dora Angelaki, Department of Anatomy and Neurobiology, Box 8108, Washington University School of Medicine, 660 South Euclid Avenue, St. Louis, MO 63110. E-mail: angelaki@thalamus.wustl.edu. Copyright (C) 2001 Society for Neuroscience $0270-6474 / 01 / 213968-18 \$ 15.00 / 0$ and eye-contra cells, respectively) exhibited distinct dynamic properties during TrVOR suppression. Eye-ipsi neurons demonstrated relatively flat dynamics that was similar to that of the majority of vestibular-only neurons. In contrast, eye-contra cells were characterized by low-pass filter dynamics relative to linear acceleration and lower sensitivities than eye-ipsi cells. In fact, the main secondary eye-contra neuron in the disynaptic RVOR pathways (position-vestibular-pause cell) that exhibits a robust modulation during RVOR suppression did not modulate during TrVOR suppression. To explain these results, a simple model is proposed that is consistent with the known neuroanatomy and postulates differential projections of sensory canal and otolith signals onto eye-contra and eye-ipsi cells, respectively, within a shared premotor circuitry that generates the VORs.

Key words: eye movement; binocular; vestibular; vestibuloocular; modeling; otolith organs; sensorimotor et al., 1994, 1996, 1997; Imagawa et al., 1995). These differences at both sensory and motor levels, as well as the existence of unique neuroanatomical connections, suggest that the sensorimotor processing of canal and otolith signals in the RVOR and TrVOR is at least partially distinct.

In the case of the RVOR, it has been well established that the most direct vestibulo-ocular connections are disynaptic, involving second-order neurons in the rostral vestibular nuclei (Richter and Precht, 1968; Baker et al., 1969; Precht et al., 1969; Schwindt et al., 1973; McCrea et al., 1987; Scudder and Fuchs, 1992). The majority of these premotor neurons carry a combination of signals that are correlated with both vestibular canal inputs (i.e., "sensory" signals) and eye movement-related activity (i.e., "motor-like" signals). The neural elements in the TrVOR are only now beginning to be identified. To date, data regarding the neural processing of otolith-ocular signals on eye movementsensitive neurons have been obtained mainly during eccentric rotations when the semicircular canals and otolith organs are simultaneously activated (McConville et al., 1996; Snyder and King, 1996; Chen-Huang and McCrea, 1999). Assumptions regarding the linear superposition of canal and otolith signals were thus required in these studies to parse out the RVOR- versus TrVOR-related contributions on individual cells.

The present study represents a first attempt to investigate directly the neural elements that participate in the dynamic transformations of the TrVOR during pure translational movements. The study focuses on the identification and quantitative comparison of translational and rotational responses of eye movementsensitive neurons in the vestibular nuclei, a subset of which represents the main premotor cells in the RVOR. During $0.5 \mathrm{~Hz}$ 
rotation, most premotor cells carry both sensory head movement and oculomotor-like signals. We demonstrate that, in contrast, during $0.5 \mathrm{~Hz}$ translation, cell responses were more closely related to the oculomotor output than to the vestibular sensory signal. Furthermore, distinct differences were observed in the sensitivity and dynamics of eye movement-sensitive neurons that code for ipsilaterally directed versus contralaterally directed eye movements (i.e., eye-ipsi vs eye-contra cells). On the basis of these results and the unique neuroanatomy of the otolith-ocular system, we propose that otolith signals join the premotor circuitry by selectively projecting onto eye-ipsi cells (as opposed to the semicircular canals that make direct projections onto eye-contra cells). Therefore, although the same subsets of neurons may participate in both reflexes, the pattern of sensory signal flow may be different for the translational and rotational components of the VORs.

\section{MATERIALS AND METHODS}

Surgical procedures and animal preparation. Data reported here were collected from three juvenile rhesus monkeys that were prepared for chronic recording of binocular eye movements and single-unit activity. In a first surgery, animals were chronically implanted with a delrin headrestrainment ring that was anchored by stainless steel screws, placed as inverted T-bolts under the skull and then secured to the ring. In addition, a delrin platform $(3 \times 3 \mathrm{~cm} ; 5 \mathrm{~mm}$ height $)$ was stereotaxically secured to the skull and fitted inside the head ring. The platform had staggered rows of holes (spaced $0.8 \mathrm{~mm}$ apart) that extended from the midline to $10 \mathrm{~mm}$ bilaterally. In separate surgeries, all animals were also implanted with dual eye coils on both eyes (cf. Angelaki, 1998; Angelaki et al., 2000a,b). In two of the animals, labyrinthine stimulating electrodes were implanted bilaterally (cf. Angelaki et al., 2000b). All surgical procedures were performed under sterile conditions in accordance with institutional and National Institutes of Health guidelines.

Eye movement recordings. Eye movements were measured with a twofield magnetic search coil system (16 inch cube; 66 and $100 \mathrm{kHz}$; CNC Engineering) that was attached to the inner gimbal of the turntable. Binocular eye movements were recorded in three dimensions. Eye coils were calibrated both before implantation as well as daily during experiments, as explained in detail elsewhere (Angelaki, 1998; Angelaki et al., 2000a,b; McHenry and Angelaki, 2000).

Experimental conditions. During experiments, the monkeys were seated in a primate chair that was secured inside the inner frame of a vestibular turntable consisting of a three-dimensional rotator on top of a $2 \mathrm{~m}$ linear sled (Acutronics, Inc.). Rotational stimulus profiles were delivered using two independently controlled rotational drives. The translational stimulus profiles were generated using the linear sled that moved in an earth-horizontal plane. Both stimulus presentation and data acquisition were controlled with custom-written scripts within the Spike2 software environment using the Cambridge Electronics Device (CED, model 1401) data acquisition system. The behavioral performance of the animal was monitored via interactions with a second "slave" computer that provided a continuous on-line TTL pulse as long as both ocular positions were maintained within $1^{\circ}$ of ideal target fixation. This "eyein-window" signal was monitored by the CED for on-line juice reward delivery and was saved for off-line analyses. Behavioral windows for each eye were calculated on-line on the basis of the geometrical relationships that should govern appropriate target fixation or ideal target stabilization for a given motion of the target and/or head movement (Angelaki et al., 2000a; McHenry and Angelaki, 2000). Juice rewards were typically given at a frequency of $\sim 1-2$ per second as long as the gaze directions of both eyes were within the specified behavioral windows.

Animals were trained to fixate a small target light that was backprojected using a laser and $x-y$ mirror galvanometer system (General Scanning) on a near screen that moved with the animal (mean vergence angle of $\sim 6.4^{\circ}$ ). For head-stationary horizontal and vertical fixations and pursuit, the galvanometer was controlled directly by using Spike2 scripts and the CED system. Because the target-laser assembly was fixed to the inner gimbal of the rotator and sled superstructure, a constant galvanometer signal (i.e., no target movement) provided a head-fixed target for the RVOR and TrVOR suppression paradigms. Earth-fixed targets during rotation and lateral translation were presented using an appropriately scaled position feedback signal from the motion delivery system to drive the galvanometers on-line during motion. Because of the underlying geometry, stabilization of a near earth-fixed target during translation elicits a slightly triangular eye velocity (see Figs. 1-3). [For an earth-fixed target during a sinusoidal translational movement trajectory, appropriate ocular angular deviations are nonsinusoidal under near viewing conditions. This is in contrast to the case of near target pursuit in which the angle of the laser target was changed sinusoidally. Thus, ocular deviations should also be approximately sinusoidal (given similar ocular-toscreen and galvanometer-to-screen distances).]

Extracellular recordings from vestibular nuclei neurons were obtained with epoxy-coated, etched tungsten microelectrodes (MicroProbe; 2-4 $\mathrm{M} \Omega$ impedance) that were inserted into the brain through a 26 gauge stainless steel guide tube (outside diameter of $457 \mu \mathrm{m}$ ). Electrodes were inserted into guide tubes and then advanced through a predrilled hole in the recording platform and manipulated vertically with a remote-control mechanical microdrive. Neural activity was amplified, filtered $(300 \mathrm{~Hz}$ to $6 \mathrm{kHz}$ ), and passed both to an audio amplifier and to a BAK Instruments dual time-amplitude window discriminator the output of which was displayed on an oscilloscope.

For each recorded cell, acceptance pulses from the BAK window discriminator were used to trigger the event channel of the CED data acquisition system that stored the time of the spike at a $10 \mu \mathrm{sec}$ resolution. In addition, the eight voltage signals from the two eye coil assemblies [four for each dual eye coil (see Angelaki, 1998)], the eye-in-window signal from the slave computer to confirm appropriate behavioral performance, the three output signals of a three-dimensional linear accelerometer (mounted on Fiberglas members that firmly attached the head ring of the animal to the inner gimbal of the rotator), and the velocity and position feedback signals from the rotator were antialias filtered $(200 \mathrm{~Hz}$; six-pole Bessel) and digitized by the CED at a rate of $833.33 \mathrm{~Hz}$ (16-bit resolution). All acquired data were stored on a personal computer for off-line analysis.

Vestibular nuclei neuron recordings. During the first experiments in each animal, the abducens nuclei were identified on the basis of the characteristic burst-tonic activity of the neurons (Fuchs and Luschei, 1970; Fuchs et al., 1988). Subsequent penetrations were concentrated in a relatively small area in the rostral part of the vestibular nucleus extending $3 \mathrm{~mm}$ posterior and $4 \mathrm{~mm}$ lateral from the center of the abducens nuclei. These areas, consisting mainly of the rostral medial vestibular nuclei (with a few penetrations extending into the caudal aspects of the superior vestibular nucleus), have been shown to contain eye movement-sensitive cells, many of which project directly to the abducens and oculomotor nuclei (McCrea et al., 1987; Scudder and Fuchs, 1992; Cullen and McCrea, 1993). In the animals that were implanted with bilateral labyrinthine stimulating electrodes, localization of the vestibular nuclei was also guided by vestibular field potentials evoked with electrical stimulation of the ipsilateral vestibular nerve $(0.1 \mathrm{msec}$ monophasic pulses; $50-400 \mu \mathrm{A}$ ).

After a vestibular nuclei neuron was isolated, a specific set of protocols was used to characterize the responsiveness of each cell. Neural responses were first recorded during both horizontal and vertical smooth pursuit $\left(0.5 \mathrm{~Hz} ; \pm 10^{\circ}\right)$, as well as during visually guided saccades to different fixation points $\left( \pm 20^{\circ}\right)$. Neural activity was subsequently recorded during $0.5 \mathrm{~Hz}\left( \pm 10^{\circ}\right)$ sinusoidal rotation in yaw and pitch with the animal upright during fixation of head-fixed and earth-fixed central targets. The axis of yaw rotations was always earth-vertical and passed through the midsagittal plane of the monkey, intersecting the line connecting the two auditory meati. The axis of pitch rotations was earthhorizontal and closely aligned with the interaural line. During smooth pursuit, stable gaze (i.e., earth-fixed target stabilization), and suppression (i.e., head-fixed target stabilization) paradigms, trained animals followed the target with smooth eye movements, whereas catch-up saccades or fast phases were usually $<1^{\circ}$. As a result, cell responses to saccadic eye movements are not clearly evident in the neural traces (see Figs. 1-4).

On the basis of their neural responses during these preliminary protocols, cells were classified into one of four groups (Scudder and Fuchs, 1992). (1) Position-vestibular-pause (PVP) and position-vestibular (PV) neurons were characterized by sensitivities to angular head velocity and to eye velocity in opposite directions such that these signals superimposed during stabilization of an earth-fixed target. PV and PVP cells included units the activities of which modulated either in-phase with ipsilateral head velocity during yaw RVOR suppression and contralaterally directed eye velocity during horizontal smooth pursuit (type I PV/PVP) or in-phase with contralateral head velocity during RVOR 
suppression and ipsilaterally directed eye velocity during smooth pursuit (type II PV/PVP). The majority of these neurons paused during saccades (PVP cells), although a number of such cells did not exhibit a clear pause (PV cells). Because the firing properties of the cells during saccades were independent of their responses during the slow eye movement protocols tested, PV neurons have been grouped together with PVP neurons in this analysis. (2) Eye-head (E-H) neurons exhibited a sensitivity to head velocity during RVOR suppression and to eye velocity during smooth pursuit in the same direction, such that the two signals opposed each other during rotation while stabilizing an earth-fixed target. This group included cells with ipsilaterally directed eye and head velocity sensitivities (Ei-Hi) as well as cells with contralaterally directed eye and head velocity sensitivities $(\mathrm{Ec}-\mathrm{Hc})$. The majority of these neurons exhibited bursts during saccades in at least one direction. (3) Burst-tonic (BT) neurons did not demonstrate modulations in firing activity during either yaw or pitch RVOR suppression but exhibited significant responses during either horizontal or vertical fixations and smooth pursuit eye movements. (4) Vestibular-only (VO) neurons included all cells that did not exhibit any slow eye movement sensitivity but modulated during either rotational or translational head movements. The present analysis focuses specifically on the translational responses of those neuron types the firing rates of which exhibited some form of correlation with the slow component of the eye movement (i.e., PV/PVP, $\mathrm{BT}$, and E-H cells), because it is a subset of these cell groups that has been shown to be the premotor neurons in the RVOR pathways (McCrea et al., 1987; Scudder and Fuchs, 1992).

All PV/PVP and E-H cells the properties of which were extensively studied during translation were also tested during RVOR suppression with the animal pitched $30^{\circ}$ nose-up and nose-down to verify that their major rotational input was from the horizontal semicircular canals. For example, the PV/PVP and E-H cells that exhibited responses of the same polarity (i.e., type I or type II) during RVOR suppression with the animal in upright, $30^{\circ}$ nose-up and $30^{\circ}$ nose-down orientations were classified as horizontal neurons. Such cells typically were also confirmed to exhibit no response modulation during pitch RVOR suppression. Cells that responded during pitch RVOR suppression and/or reversed response polarity (i.e., from type I to type II or vice versa) for yaw RVOR suppression in nose-up versus nose-down orientations were classified as vertical cells. All horizontal E-H and PV/PVP neurons also exhibited larger response modulations during horizontal pursuit and fixations as compared with vertical pursuit and fixations. For BT cells, the distinction between horizontal and vertical was based exclusively on their eye movement sensitivities.

Head translation protocols. This study primarily focused on the characterization of the steady-state sinusoidal responses of PV/PVP, BT, and E-H neurons at $0.5 \mathrm{~Hz}$ where both pursuit and VOR suppression performance are excellent. After a cell was satisfactorily characterized on the basis of its responses for various combinations of visual and head rotational stimuli, the following paradigms were used to characterize its response to translational movements.

(1) All neurons were tested during lateral and fore-aft translation at $0.5 \mathrm{~Hz}( \pm 0.2 \mathrm{~g})$ while the animal viewed a near head-fixed central target (TrVOR suppression). If neural isolation was maintained, the spatiotemporal properties of the cell were also tested during TrVOR suppression at different frequencies and for different translation directions $(0.16 \mathrm{~Hz}$, $0.1 \mathrm{~g} ; 0.3-2 \mathrm{~Hz}, 0.2 \mathrm{~g}$ ). This was done by reorienting the animal relative to the linear track to achieve translations in different directions (i.e., translation directions in between the interaural and naso-occipital axes). The animal was repositioned in steps of $30^{\circ}$, through a maximum angle of $180^{\circ}$, as long as cell isolation was maintained.

(2) In approximately one-third of the neural population, responses were also examined during lateral translation while the animal fixated an earth-fixed near, central target $(0.5 \mathrm{~Hz}$ at $\pm 0.05 \mathrm{~g} ; \pm 4.8 \mathrm{~cm})$ eliciting an eye movement of approximately $\pm 10^{\circ}$. This translational stimulus was chosen specifically to ensure that the ocular deviations required to maintain target fixation were of the same peak amplitude and vergence angle as those elicited during the horizontal smooth pursuit paradigm $\left(0.5 \mathrm{~Hz} ; \pm 10^{\circ} ; 6.4^{\circ}\right.$ mean vergence angle; performed as part of the "classification" protocol).

The rationale behind the latter experimental protocol was to enable a direct comparison of central neuron responses during rotation or translation with those during pursuit in an assumption-free manner. In particular, head movement stimuli were chosen such that they would elicit eye movements of amplitude, frequency, and mean vergence angle similar to those during pursuit. In previous studies, estimates of the degree to which different signals contribute to the response of a cell have typically been made by parsing out cell-firing rates according to their eye position, eye velocity, and head movement sensitivities. However, these sensitivity values were often isolated during different experimental paradigms without keeping stimulus frequency and viewing distance constant. Our experimental protocol was specifically chosen to avoid making assumptions about the independence of eye movement-related activity on viewing distance and frequency. Furthermore, this approach allowed us to avoid the particular problems associated with parsing out signal components on eye-head cells, which often exhibit highly nonlinear sensitivities to ocular deviations (Scudder and Fuchs, 1992; Lisberger et al., 1994; Chen-Huang and McCrea, 1999).

The use of sinusoidal stimulation at $0.5 \mathrm{~Hz}$ to study stable gaze responses during translation deserves further comment because of the fact that this is a low-frequency stimulus for the TrVOR, which is robust in the absence of visual feedback only at relatively high frequencies $(>0.5$ $\mathrm{Hz}$ ) (Paige and Tomko, 1991a; Telford et al., 1997). In other words, the observed eye movements during $0.5 \mathrm{~Hz}$ TrVOR stable gaze are presumably primarily visually evoked (i.e., pursuit responses). It is for this reason that the $0.5 \mathrm{~Hz}$ stimulus frequency is in fact optimal for the goals of the present study. Specifically, although the TrVOR in darkness at 0.5 $\mathrm{Hz}$ is small, both primary otolith afferent and central otolith-only neurons do indeed exhibit robust responses to translation at this frequency (Angelaki and Dickman, 2000). Hence, an investigation of cell responses at $0.5 \mathrm{~Hz}$ under both stable gaze and suppression conditions was expected to provide a clear means of addressing the degree to which neural activities reflect motor versus sensory vestibular information.

Data analyses. All data analyses were performed off-line using Matlab (Mathworks, Inc.). Eye positions were calibrated and expressed as threedimensional rotation vectors, as described in detail elsewhere (Angelaki, 1998; Angelaki et al., 2000a). During many of the recordings in one of the animals, the torsion coil of the left eye was broken. In these cases, only right eye movements were included in the illustrations (e.g., see Figs. 1-2), although behavioral control (i.e., verification of appropriate performance and reward) was based on binocular eye position. Saccades were detected and marked using a semiautomated algorithm that detects saccades by thresholding the second derivative of the eye velocity vector. For the neural activity, unit clock values were converted to an instantaneous firing rate that was computed as the inverse of interspike interval and assigned to the middle of the interval. For each experimental run, neural data were first desaccaded using a window that extended from 50 msec before to 100-200 msec after each saccade (Scudder and Fuchs, 1992). Fixation data and multiple linear regression analyses were used to estimate the eye position sensitivity of the neuron.

Firing rates from 3 to 20 cycles were then folded into a single cycle (no averaging was performed). Only portions of data in which the positions of both eyes were within $\pm 1^{\circ}$ of the target were included in the folding and further analyses. The peak amplitude and phase of eye and head velocity as well as neural firing rates during translation, rotation, and pursuit were then determined by fitting a sine function (first and second harmonics and a DC offset) to the overlaid data using a nonlinear least-squares algorithm based on the Levenberg-Marquardt method. Portions of the cycle in which neurons were silent were excluded from the least-squares optimization. Whenever neural response gains were estimated, units were spikes $\cdot \mathrm{sec}^{-1}$ per degree $\cdot \sec ^{-1}$ for rotation and pursuit and spikes $\cdot \mathrm{sec}^{-1} \cdot$ gravity $^{-1}$ (with $g=9.81 \mathrm{~m} / \mathrm{sec}^{2}$ ) for translation (the latter was chosen for a direct comparison of the TrVOR suppression responses of eye movement-sensitive cells with those reported previously for otolith afferent and vestibular-only neurons). Phase was expressed as the difference (in degrees) between peak neural activity and peak head (for rotation and translation) or eye (for pursuit) velocity.

A cell was considered to exhibit a significant response to TrVOR suppression, RVOR suppression, or smooth pursuit if the following criteria were met: (1) Harmonic distortion was $<35 \%$ during rotation, translation, and/or pursuit in at least one stimulus direction. (2) Response gain was greater than a minimum of $0.10 \mathrm{spikes} \cdot \mathrm{sec}^{-1}$ per degree $\cdot \sec ^{-1}$ for head rotation and pursuit and $>20 \mathrm{spikes} \cdot \mathrm{sec}^{-1} \cdot g^{-1}$ for translation along the directions of minimum harmonic distortion. For the cells that fulfilled these two criteria, a clear modulation in firing rate was also heard through the audio amplifier.

The spatial tuning characteristics of the cells that exhibited a significant modulation during TrVOR suppression were evaluated by fitting a two-dimensional spatiotemporal model to the gain and phase data as a function of stimulus direction (for details, see Angelaki, 1991; Angelaki and Dickman, 2000). This model is an extension of the one-dimensional, 
cosine-tuning equations that are often used to describe the spatial tuning of vestibular signals. The two-dimensional spatiotemporal technique differs from the traditional one-dimensional cosine approach in that it considers the dependence of both gain and phase on spatial orientation and provides the ability to describe neural responses in cases in which the response of the cell in the minimum sensitivity direction is nonzero (the one-dimensional approach assumes a zero response in the direction of minimum sensitivity). The ratio of the response gain of the cell in the minimum sensitivity direction over that in the maximum sensitivity direction is referred to as the tuning ratio and provides a measure of the spatial specificity of the cell. The majority of VO neurons in the rostral vestibular nuclei of primates have been shown recently to exhibit twodimensional spatiotemporal coding properties and to have tuning ratios $>0.1$ (Angelaki and Dickman, 2000).

Bode plots were constructed to compare the response dynamics among the different cell groups during TrVOR suppression over a frequency range of $0.16-2 \mathrm{~Hz}$. Only portions of each trial in which the animal maintained fixation within $1^{\circ}$ of the target (based on the eye-in-window signal) and for which peak eye velocity was $<2 \%$ sec were included in the analysis. For a convenient visual inspection of differences in dynamics among different groups of neurons and to preserve the appropriate frequency response shape despite averaging over multiple neurons with different sensitivities, gains at all frequencies were normalized to the sensitivity of the cell at $0.5 \mathrm{~Hz}$ (see Fig. 7). That is, before averaging, all cell gains were scaled to unity at $0.5 \mathrm{~Hz}$, whereas at other frequencies, cell gains were larger or smaller than unity depending on whether sensitivities were larger or smaller than those at $0.5 \mathrm{~Hz}$. Statistical comparisons were based on ANOVA.

Histology. At the completion of all recording experiments, the animals were deeply anesthetized (Pentobarbital sodium) and perfused transcardially with a $2 \%$ paraformaldehyde and $2 \%$ glutaraldehyde solution. The brain was removed, sectioned $(80 \mu \mathrm{m})$, and counterstained (alternate sections with cresyl violet and Weil). An approximate recording location map was reconstructed for each animal, using the penetration records and identified location of the abducens nucleus as guides. The exact recording sites could not be verified on the basis of histological examination.

Model simulations. A model (see Fig. 10 for illustration) was explored as a means of interpreting both our current data and the differences in the short-latency neuroanatomy of the canal-ocular versus otolith-ocular pathways. The model represents an extension of that proposed by Green and Galiana (1998) to incorporate an additional eye-ipsi cell type. The basis for the model and its key characteristics are described in detail in Results. Model predictions were investigated analytically by deriving Laplace transform descriptions of ocular and central responses (see Appendix). By use of these analytical descriptions, Bode plots describing response gains and phases were constructed using Matlab (Mathworks, Inc.; version 5.2). In addition, the model was implemented with the Matlab dynamic simulation environment Simulink (version 2.2). Model simulations were performed using a Runge-Kutta integration routine with time steps of $0.01 \mathrm{sec}$. Simulated responses were cross-checked with analytical predictions to ensure consistency.

\section{RESULTS}

\section{Experimental data}

General comparison of responses to head rotation versus translation

Of 224 neurons that were characterized in the rostral vestibular nuclei of three animals, 130 exhibited eye movement-related activity and were isolated long enough to complete the classification protocol. On the basis of neural firing rates during visual fixation, smooth pursuit, and yaw or pitch rotations while an animal fixated a head-fixed target, neurons were classified into three groups (see Materials and Methods for a more detailed description). These included PV/PVP, BT, and E-H cells (Keller and Kamath, 1975; Tomlinson and Robinson, 1984; Scudder and Fuchs, 1992; Cullen and McCrea, 1993; Lisberger et al., 1994). Typical example responses from each group of eye-contra cells (i.e., type I PV/PVP, BT, and Ec-Hc neurons) have been illustrated (see Figs. 1-3). Two example responses from eye-ipsi cells (i.e., type II PV/PVP and Ei-Hi neurons) are also illustrated (see Fig. 4).

Type I PV/PVP neurons increased their firing rates with contralaterally directed eye velocity during horizontal pursuit and with ipsilaterally directed head velocity during RVOR suppression (Fig. 1). During yaw rotation while an animal fixated a near earth-fixed target, these two signals superimposed. When the animal was translated laterally while stabilizing an earth-fixed target, all type I PV/PVP neurons exhibited a robust response modulation and increased their firing rates during ipsilateral translation. Hence, their responses to translation during earthfixed target viewing were consistent with those during head rotation (i.e., the cells demonstrated activities appropriate to drive contralaterally directed eye movements). A clear distinction could be made between type I PV/PVP cell responses to head rotation and translation when their activities were compared during head-fixed target viewing. Whereas type I PV/PV Ps demonstrated a robust modulation in the absence of eye movements during head rotation, these cells exhibited no response during TrVOR suppression (Fig. 1, Table 1). Type II PV/PVPs (i.e., neurons with ipsilateral eye movement and contralateral head velocity sensitivities), however, behaved differently from type I PV/PVP cells. Specifically, most type II PV/PVP neurons exhibited clear response modulations during both head rotation and translation under head-fixed target stabilization conditions (see Fig. 4A, Table 1).

Neurons that were classified as BT behaved comparably during head translations and rotations, apparently coding always for eye movement-related signals, regardless of the source of sensory input. For example, the cell illustrated in Figure 2 was excited during contralaterally directed eye movements but did not respond during RVOR suppression. Similarly, none of the BT neurons encountered exhibited a consistent response modulation during lateral TrVOR suppression. As typical for all contralateral BT cells, the neuron exhibited an increase in firing rate during both ipsilaterally directed head rotations and translations while an earth-fixed target was stabilized.

Eye-head neurons exhibited all the salient features that have been described previously in primates, including eye and head velocity sensitivities in the same direction, nonlinear firing rate/ eye position curves, and inconsistent behavior during saccadic eye movements (Scudder and Fuchs, 1992; Lisberger et al., 1994; Chen-Huang and McCrea, 1999). Ec-Hc cells increased their firing rates with contralaterally directed eye velocity during horizontal pursuit and contralaterally directed head velocity during yaw RVOR suppression (Fig. 3). Ipsilateral E-H cells were excited for ipsilaterally directed eye and head deviations (Fig. 4B). In general, response sensitivity and phase during rotation while an earth-fixed target was stabilized was variable. Some neurons exhibited a similar phase relationship to the head velocity stimulus for both head-fixed and earth-fixed target stabilization conditions during rotation (e.g., the cell in Fig. 3, which increased its firing rate with contralateral head velocity). However, other E-H cells exhibited a phase reversal during RVOR suppression relative to their direction of modulation under earth-fixed target viewing conditions.

In contrast to type I PV/PVP cells that exhibited negligible responses during translation while a head-fixed target was stabilized, approximately one-half of the recorded E-H cells exhibited a clear (although often small) modulation in their firing rates when the animal suppressed his eye movements (Figs. 3, 4B; TrVOR suppression; see Table 1). Notably, E-H cells did not 
Type I PVP

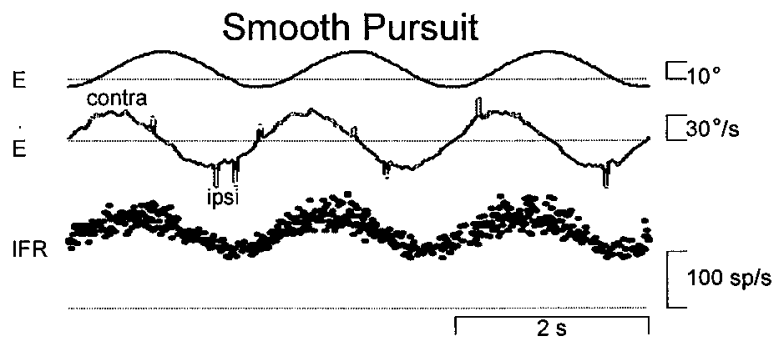

RVOR suppression
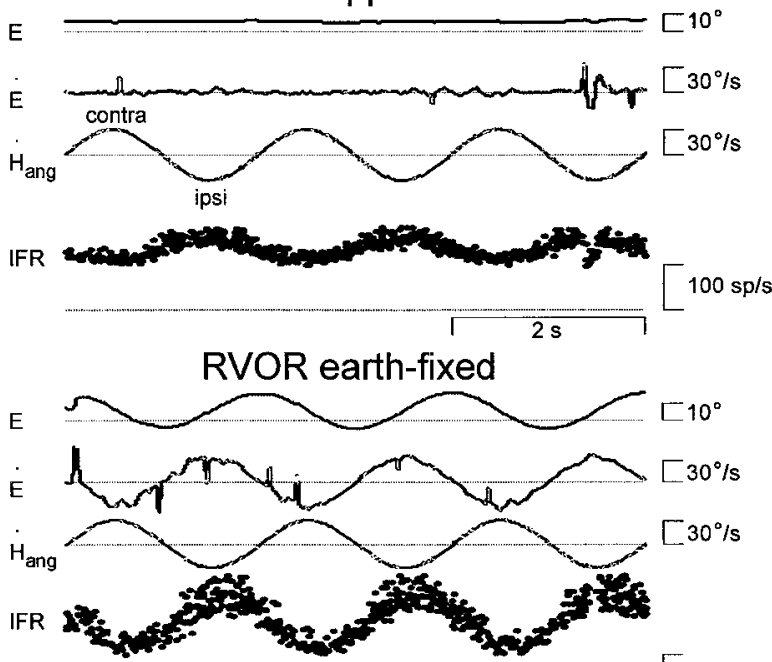

$100 \mathrm{sp} / \mathrm{s}$

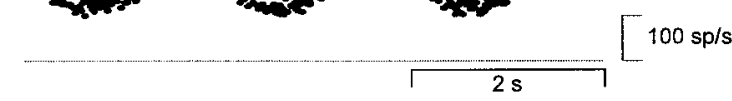

TrVOR suppression

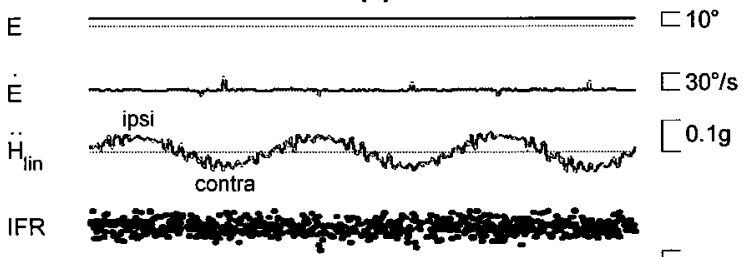

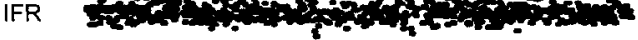

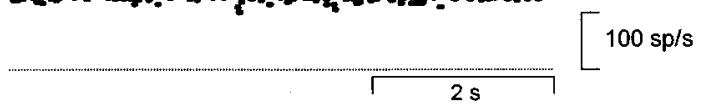

TrVOR earth-fixed

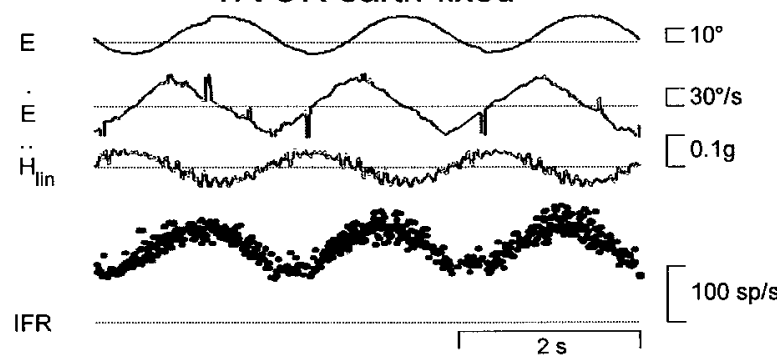

Figure 1. Responses of a horizontal type I position-vestibular-pause $(P V P)$ neuron during smooth pursuit, yaw rotation, and lateral translation. During head movement, subjects stabilized either a head-fixed (RVOR or TrVOR suppression) or an earth-fixed $\left(0.5 \mathrm{~Hz}, \pm 10^{\circ}\right.$ ocular deviations) target. The pause of the cell is only evident for one of the largest saccades during RVOR suppression (fast changes in eye position are usually $\ll 1^{\circ}$ during all experimental paradigms). From top to bottom, right eye position $(E)$, right eye velocity $(\dot{E})$, stimulus (head velocity, $\dot{H}_{\text {ang }}$ for rotation and head acceleration, $\ddot{H}_{\text {lin }}$ for translation), and instantaneous firing rate $(I F R)$ of the neuron are shown. RVOR, Rotational vestibulo-ocular reflex; $s p$, spikes; $\operatorname{Tr} V O R$, translational VOR.
Table 1. Patterns of response modulation during TrVOR suppression

\begin{tabular}{|c|c|c|c|c|c|}
\hline & & $\mathrm{Yes}^{a}$ & $\mathrm{No}^{a}$ & $\begin{array}{l}\text { Total number } \\
\text { of neurons }\end{array}$ & $\begin{array}{l}\text { Percentage } \\
\text { of yes }\end{array}$ \\
\hline \multicolumn{2}{|c|}{ Hor $^{b}$ PV/PVP - type I } & 0 & 18 & 25 & $0 \%$ \\
\hline & - type II & 9 & 2 & 13 & $82 \%$ \\
\hline \multicolumn{2}{|c|}{ Vertical PV/PVP } & 0 & 7 & 8 & $0 \%$ \\
\hline \multirow[t]{2}{*}{ Hor E-H } & -Ec-Hc & 7 & 2 & 14 & $78 \%$ \\
\hline & - Ei-Hi & 6 & 8 & 18 & $43 \%$ \\
\hline \multicolumn{2}{|c|}{ Vertical E-H } & 1 & 20 & 30 & $5 \%$ \\
\hline \multicolumn{2}{|c|}{ Burst-Tonic or Tonic } & 0 & 22 & 22 & $0 \%$ \\
\hline \multicolumn{2}{|c|}{ Total } & 23 & 79 & 130 & $23 \%$ \\
\hline
\end{tabular}

${ }^{a}$ Number of neurons from each group that did (Yes) or did not (No) exhibit a systematic response modulation during TrVOR suppression. Cells were considered to modulate if their sensitivity along either the lateral or the fore-aft axis at either 0.5 or $2 \mathrm{~Hz}$ was $>20$ spikes $\cdot \mathrm{sec}^{-1} \cdot \mathrm{g}^{-1}$ with harmonic distortion $<35 \%$. Cells that were not sufficiently tested or that had ambiguous responses have been included only in the Total number of neurons column but not in the calculation of the percentage values.

${ }^{b}$ Hor, Horizontal.

always hold the same relationship to head/eye movement during rotations and translations. Specifically, neurons sensitive to contralateral rotation under head-fixed target stabilization conditions could be excited during ipsilateral translation (and vice versa). Moreover, despite the inconsistent behavior of E-H cells during head rotation under stable gaze conditions (whereby response phase was not always consistent with the eye movement preference of the cell during head-stationary pursuit), their responses to translation during earth-fixed target viewing were always appropriate to drive contralaterally (for Ec-Hc cells) or ipsilaterally (for Ei-Hi cells) directed eye movements. With the exception of one vertical eye-head cell, none of the cells that were classified as having predominately vertical eye and head movement sensitivities during pursuit and rotation modulated their firing rates during TrVOR suppression.

In summary, all eye movement-related cell types examined demonstrated activities during translation (but not necessarily rotation) while an animal viewed an earth-fixed target that were appropriately directed for their respective eye movement preference. Notably, however, during TrVOR suppression only a subpopulation of type II PV/PVP and E-H cells demonstrated a significant modulation, suggesting that distinct cell types may receive more direct sensory otolith signals. Thus, responses under both suppression and stable gaze conditions were examined in more detail to test the hypothesis that there are differences among eye-ipsi and eye-contra PV/PVP and E-H neurons in terms of their locations within the pathways underlying the transformation of sensory information into oculomotor signals in the TrVOR.

\section{Responses during TrVOR suppression}

As outlined in the previous section, only a few cells (nine type II PV/PVP, seven Ec-Hc, six Ei-Hi, and one vertical E-H; see Table 1) exhibited a clear and consistent response modulation during 0.5 and/or $2 \mathrm{~Hz}$ TrVOR suppression. The mean ( $\pm \mathrm{SD}$ ) sensitivity relative to translational acceleration for all TrVOR suppressionsensitive cells at $0.5 \mathrm{~Hz}$ was $85 \pm 82 \mathrm{spikes} \cdot \mathrm{sec}^{-1} \cdot g^{-1}(n=18$; range of $\left.21-266 \mathrm{spikes} \cdot \mathrm{sec}^{-1} \cdot g^{-1}\right)$. These suppression responses were similar in amplitude to those of VO central cells and primary otolith afferents tested under identical experimental conditions (Angelaki and Dickman, 2000). For Ei-Hi cells, no correlation was observed between cell sensitivities to head movement 


\section{Burst-Tonic}

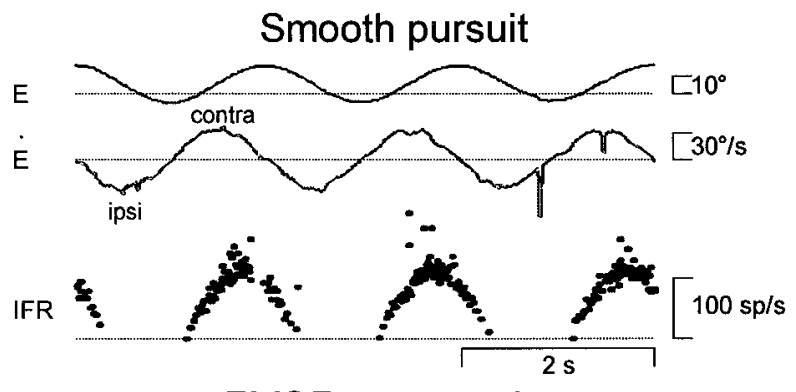

RVOR suppression
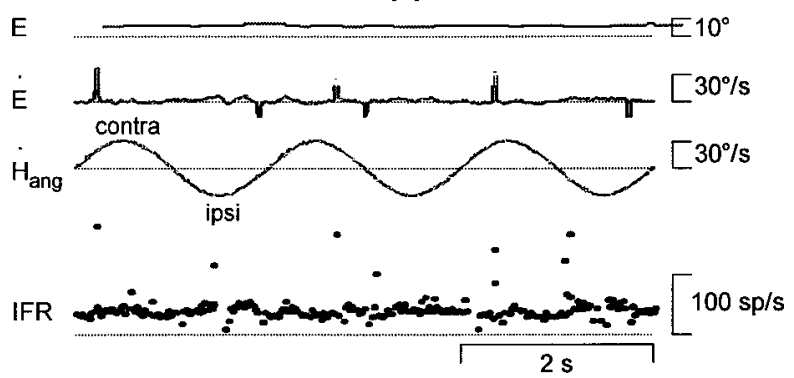

RVOR earth-fixed

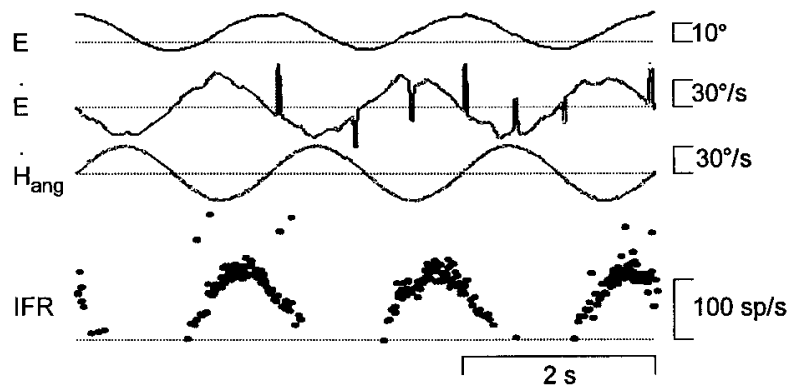

TrVOR suppression

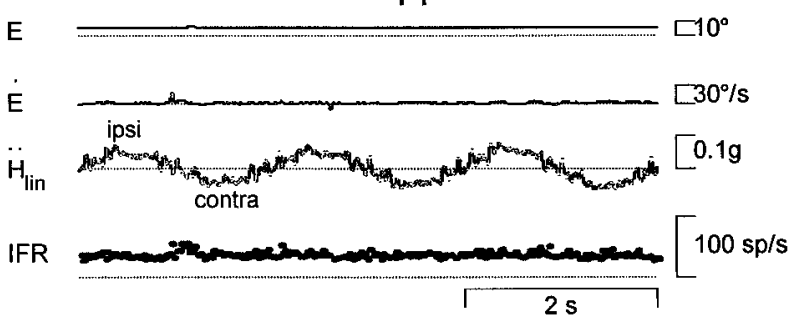

TrVOR earth-fixed

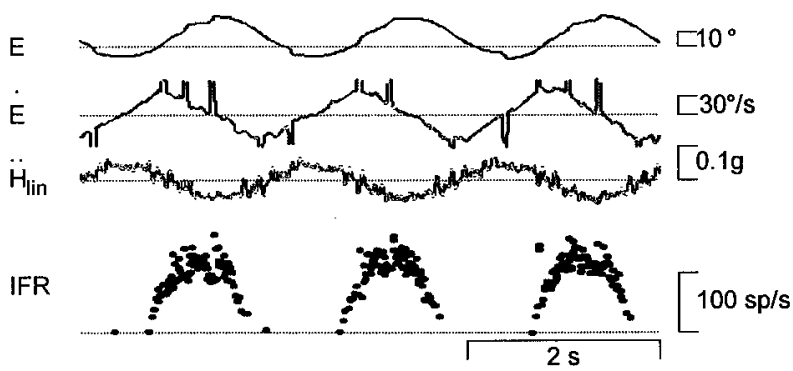

Figure 2. Responses of a horizontal burst-tonic (BT) cell with contralateral eye movement sensitivity during horizontal smooth pursuit, head rotation, and translation. During head movement, subjects stabilized either a head-fixed (RVOR or TrVOR suppression) or an earth-fixed ( 0.5 $\left.\mathrm{Hz}, \pm 10^{\circ}\right)$ target. See Figure 1 legend for other abbreviations.

\section{Eye-Head (Ec-Hc)}

\section{Smooth pursuit}
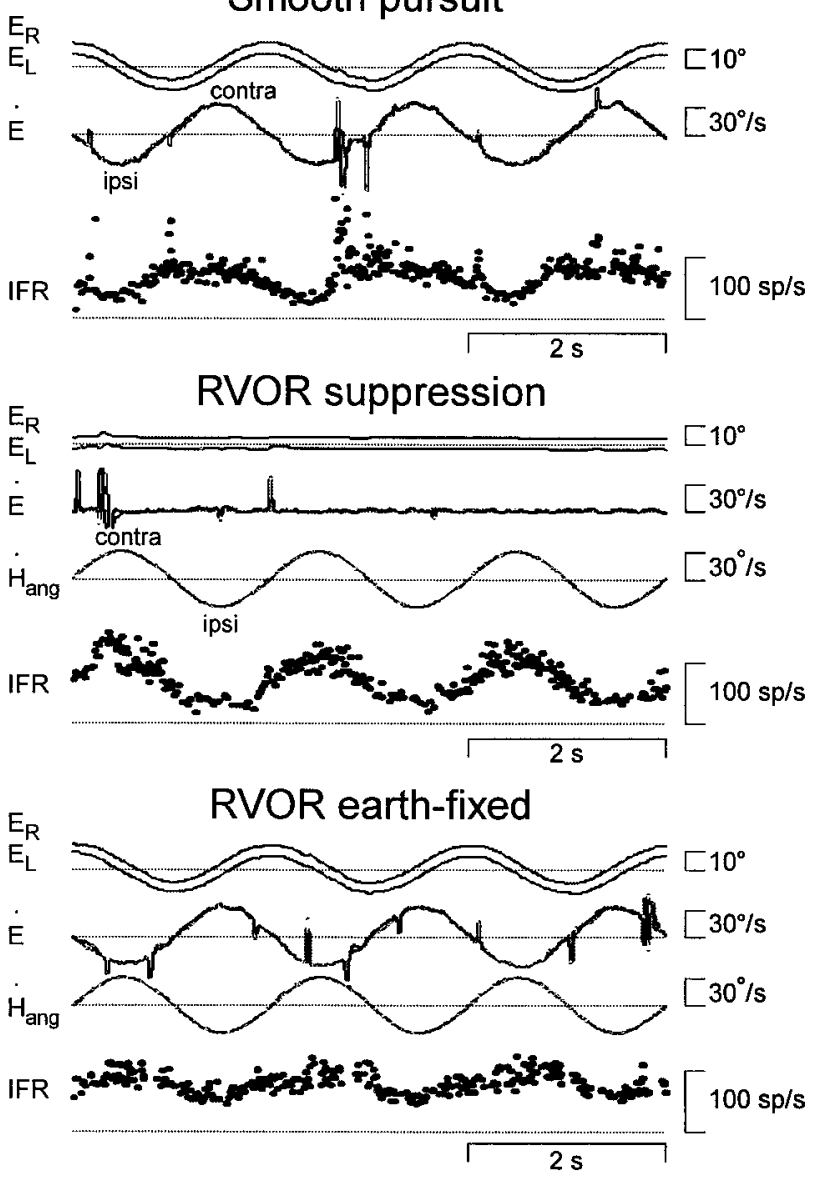

TrVOR suppression

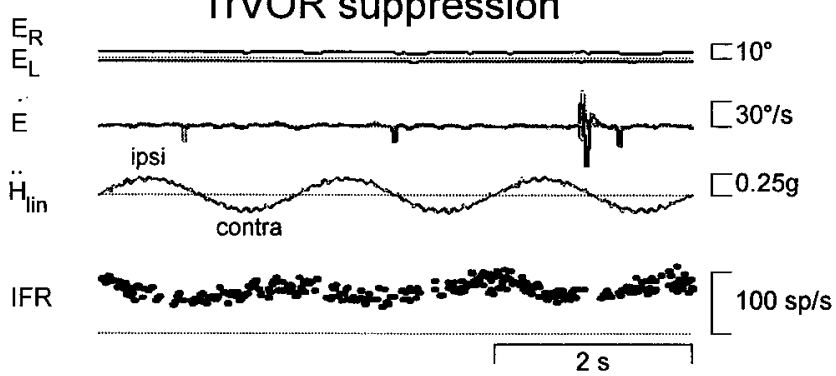

TrVOR earth-fixed

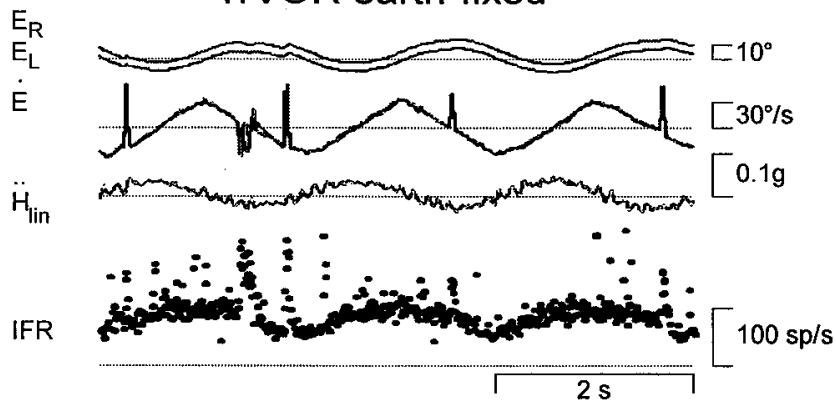

Figure 3. Responses of a horizontal eye-head cell with contralateral eye movement sensitivity $(E c-H c)$ during horizontal smooth pursuit, head rotation, and translation. During head movement, subjects stabilized either a head-fixed (RVOR or TrVOR suppression) or an earth-fixed (0.5 $\left.\mathrm{Hz}, \pm 10^{\circ}\right)$ target. See Figure 1 legend for other abbreviations. 


\section{A Type II PVP}
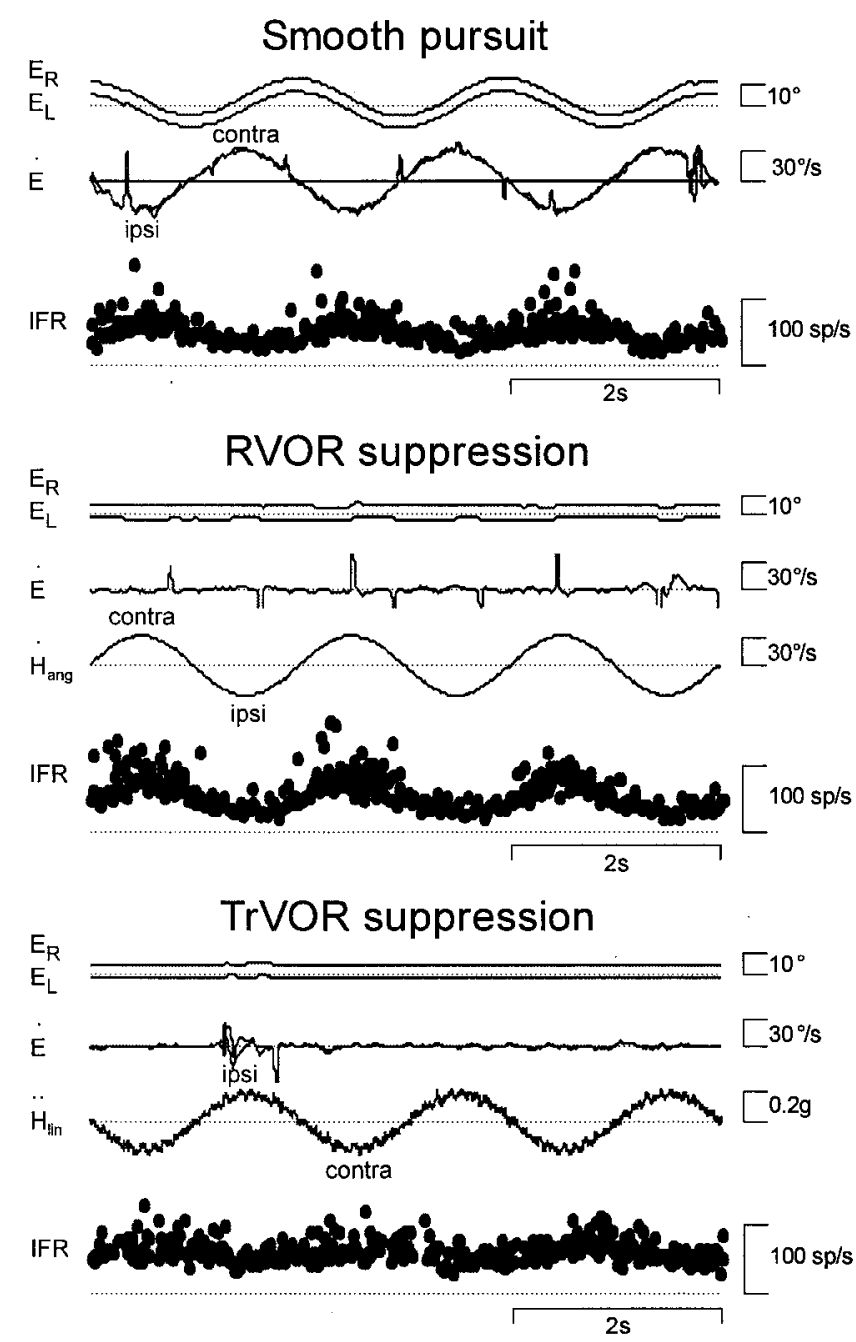

B Eye-Head (Ei-Hi)
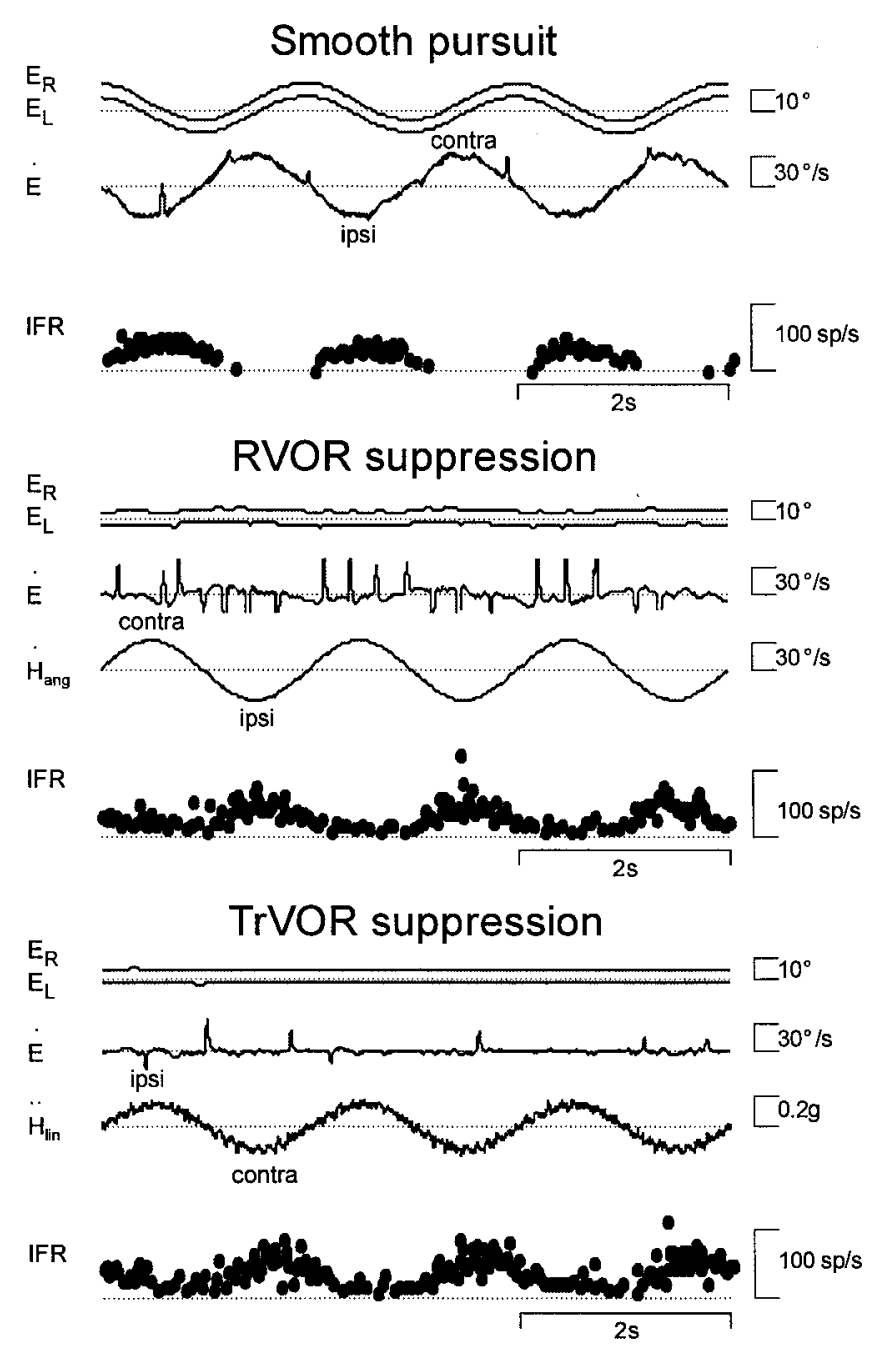

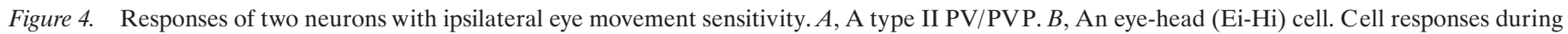
horizontal smooth pursuit, RVOR suppression, and TrVOR suppression $(0.5 \mathrm{~Hz})$ are shown. See Figure 1 legend for abbreviations.

during TrVOR suppression and RVOR suppression $\left(R^{2}=0.04\right)$. For Ec-Hc and type II PV/PVP neurons, a weak correlation existed $\left(R^{2}=0.54\right.$ and 0.60 , respectively).

Figure $5 A$ illustrates the cell responses during lateral TrVOR suppression at $0.5 \mathrm{~Hz}$, in which response phase has been described by a polar angle and response sensitivity has been described by the amplitude in the radial direction. In general, response phase during lateral TrVOR suppression varied widely among neurons, with the majority of cells exhibiting responses that either led or lagged contralaterally directed head velocity (Fig. $5 A$ ). Furthermore, the ipsilateral/contralateral preference of neurons was not always identical during RVOR and TrVOR suppression. For example, most Ei-Hi cells that responded with ipsilaterally directed sensitivities to head rotation during RVOR suppression increased their activities for contralaterally directed head velocity during TrVOR suppression. In fact, there was a general trend for all groups of cells that responded during TrVOR suppression to be excited during contralaterally directed head movement (Fig. 5A). Hence, the E-H cell classification during head rotation (i.e., in terms of the sensitivity of the cell to eye velocity during pursuit and to head velocity during suppression) does not in general extend to their responses during lateral translation.

The average sensitivity and phase for each group of cells during lateral TrVOR suppression at 0.5 and $2 \mathrm{~Hz}$ are compared in Figure $5 B$ (solid and open symbols, respectively). Eye-contra neurons (i.e., Ec-Hc) differed from eye-ipsi neurons (i.e., Ei-Hi and type II PV/PVP) in several respects. First, there were significant differences both in terms of sensitivity and phase between eyecontra and eye-ipsi cell responses $\left[F_{(1,33)}=6.05\right.$ (gain), $=6.41$ (phase); $p<0.05]$. Specifically, eye-contra cells exhibited lower sensitivities and smaller leads relative to linear velocity than did eye-ipsi cells. Second, there was a significant decrease in the sensitivity of eye-contra (but not in the sensitivity of eye-ipsi) cells between 0.5 and $2 \mathrm{~Hz}\left[F_{(1,10)}=6.1 ; p<0.05\right]$. The only difference between type II PV/PVP and Ei-Hi neurons was a tendency for the former to have larger phase leads relative to contralaterally directed head velocity $\left[F_{(1,21)}=4.4 ; p=0.05\right]$.

A subpopulation of the cells that responded during TrVOR suppression was also tested during linear motion along different 


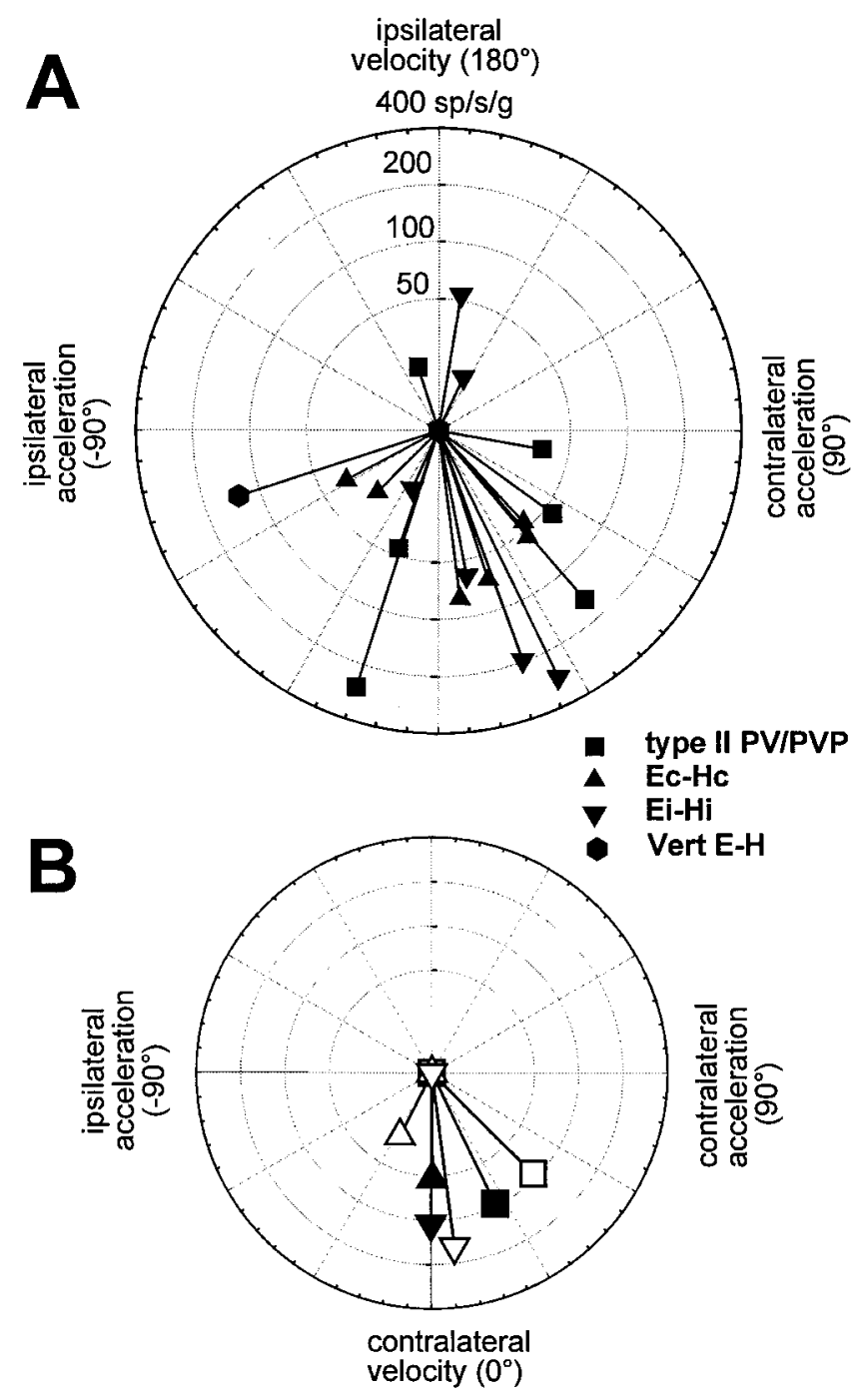

Figure 5. Response sensitivity and phase during lateral TrVOR suppression are plotted in polar coordinates for the horizontal type II PV/PVP, Ec-Hc, and Ei-Hi cells and one vertical E-H cell that exhibited clear response modulations during stabilization of a head-fixed target. $A$, Individual neuron responses at $0.5 \mathrm{~Hz}( \pm 0.2 \mathrm{~g})$ are shown. $B$, Average responses for the type II PV/PVP, Ec-Hc, and Ei-Hi neurons are compared for 0.5 and $2 \mathrm{~Hz}$ translational stimuli (solid and open symbols, respectively). Sensitivity has been expressed in spikes $\cdot$ second $^{-1} \cdot$ gravity $^{-1}(g=9.81$ $\mathrm{m} / \mathrm{sec}^{2}$ ). Phase has been expressed relative to contralaterally directed head velocity. Vert, Vertical. See previous figure legends for other abbreviations.

directions in the horizontal plane (usually six positions spaced every $30^{\circ}$, including lateral and fore-aft motion) to investigate their spatial-tuning properties. For all heading directions, the animal fixated a central head-fixed target and suppressed the generation of compensatory eye movement. The direction of maximum sensitivity, as well as the gain and phase of the neuron along the maximum sensitivity direction, was then computed by fitting a two-dimensional spatiotemporal model to the sensitivity and phase of each cell as a function of heading direction (see Materials and Methods) (for details, see also Angelaki et al., 1992; Angelaki and Dickman, 2000). Approximately one-half of the cells that responded during TrVOR suppression exhibited two-dimensional spatial-tuning properties and ratios of minimum over maximum sensitivity that were $>0.1$ (Angelaki, 1991; Angelaki et al., 1992; Angelaki and Dickman, 2000).

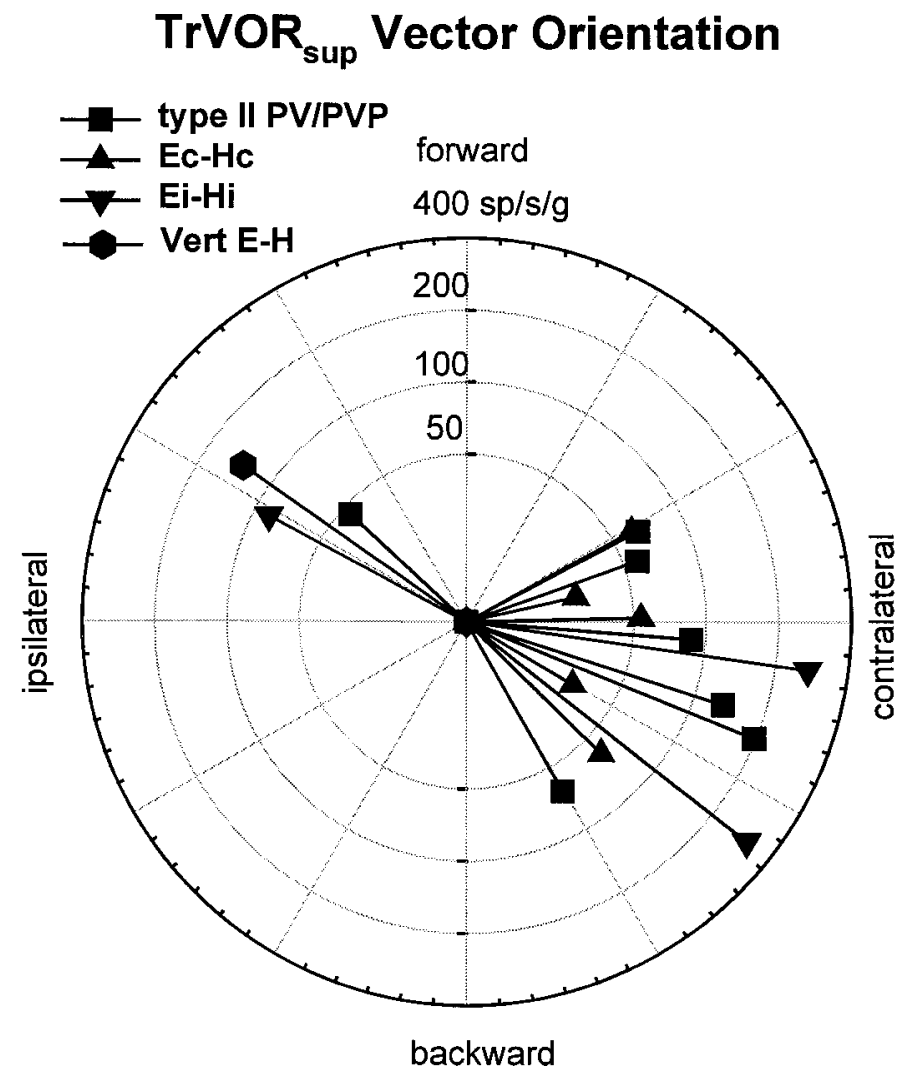

Figure 6. Distribution of maximum sensitivity vector orientations during translations in the horizontal plane for the horizontal type II PV/PVP, $\mathrm{Ec}-\mathrm{Hc}$, and Ei-Hi cells and one vertical E-H cell that exhibited clear response modulations during TrVOR suppression at $0.5 \mathrm{~Hz}$. Vector sensitivity is expressed in spikes $\cdot$ second ${ }^{-1} \cdot$ gravity $^{-1}\left(g=9.81 \mathrm{~m} / \mathrm{sec}^{2}\right)$. sup, Suppression. See previous figure legends for other abbreviations.

The distribution of maximum sensitivity vectors of those neurons that were tested for multiple translational directions has been plotted in Figure 6. As expected on the basis of the phase of the responses during lateral motion (Fig. 5), the majority of vectors were directed contralaterally [i.e., similar to the majority of primary otolith afferents and central vestibular-only neurons (Angelaki and Dickman, 2000)]. This appeared to be true for all types of responsive cells, independently of whether they exhibited ipsilaterally or contralaterally directed eye movement sensitivities or whether they were excited for ipsilaterally or contralaterally directed rotation during RVOR suppression.

The average response phase of the neurons along their maximum sensitivity direction hovered between velocity and acceleration throughout the frequency range that animals suppressed their TrVOR (0.16-2 Hz; Fig. 7). All ipsilateral eye movement cells (i.e., type II PV/PVP and Ei-Hi; Fig. 7, squares and inverted triangles) exhibited response dynamics that resembled those of "high-pass" or "flat" otolith-only VO cells [Fig. 7, dashed lines are means of the two VO groups; replotted from Angelaki and Dickman (2000)]. Accordingly, average response sensitivity (expressed in spikes $\cdot$ second $^{-1} \cdot$ gravity $^{-1}$ ) increased slightly with frequency. The response phase tended to either remain flat or demonstrate an increased lead relative to head velocity as a function of frequency.

In contrast to the dynamic properties of the eye-ipsi neurons (i.e., type II PV/PVP and Ei-Hi cells), the few Ec-Hc cells that exhibited clear responses during TrVOR suppression and were 

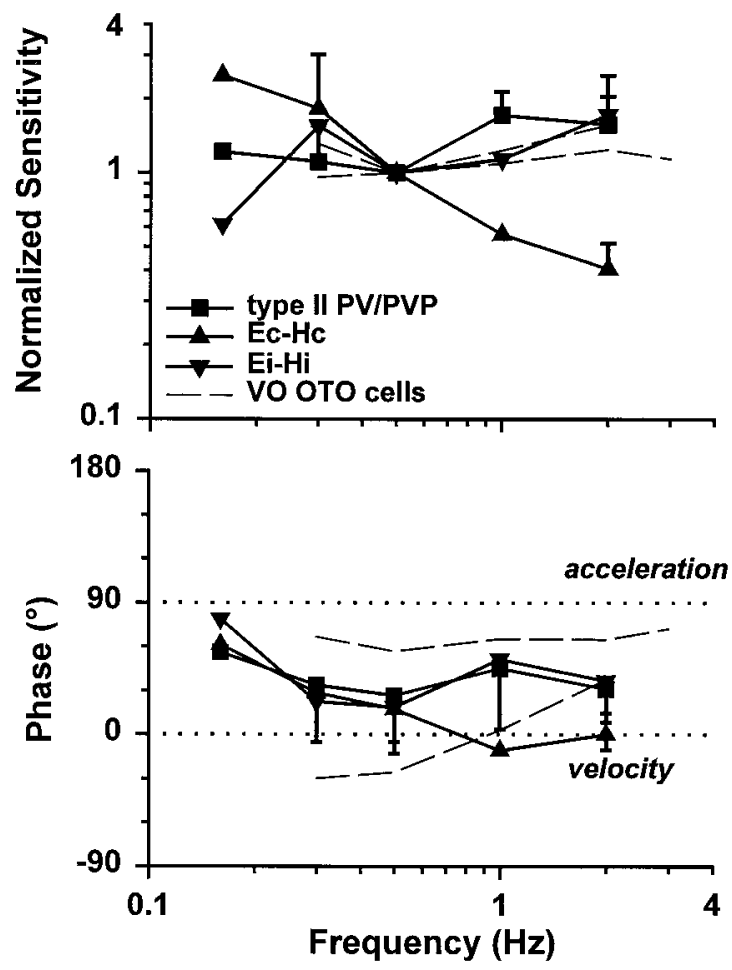

Figure 7. Response dynamics of eye-ipsi and eye-contra cells during TrVOR suppression. The mean $( \pm \mathrm{SD})$ response sensitivity (top) and phase (bottom) for translation in the maximum sensitivity direction are plotted as a function of frequency for five type II PV/PVP, two Ec-Hc, and four Ei-Hi cells. Dashed lines are used to illustrate the mean sensitivity and phase from two groups of otolith-only (i.e., not eye movementsensitive) neurons that exhibited high-pass and flat dynamics and were recorded rostrally in the vestibular nuclei, in the vicinity of the eye movement cells of the present study (Angelaki and Dickman, 2000). Phases of $0^{\circ}$ (linear velocity) and $90^{\circ}$ (linear acceleration) are indicated with dotted horizontal lines. Sensitivities are expressed in spikes $\cdot$ second $^{-1} \cdot$ gravity $^{-1}\left(g=9.81 \mathrm{~m} / \mathrm{sec}^{2}\right)$ and normalized to a gain of unity at $0.5 \mathrm{~Hz}$ before the calculation of each average. Phase values were expressed relative to translational head velocity in the interval of -90 to $+90^{\circ}$, independently of whether the firing rate of a given cell increased for ipsilaterally or contralaterally directed head movements. VO OTO, Vestibular-only otolith neurons. See previous figure legends for other abbreviations.

tested at several frequencies and for different movement directions were characterized by low-pass filter dynamics. Specifically, response sensitivity declined, and phase leads relative to head velocity decreased with frequency (Fig. 7, triangles). Decreases in gain and phase lead with frequency between 0.5 and $2 \mathrm{~Hz}$ were also observed for all responsive Ec-Hc neurons, as illustrated previously in Figure $5 B$.

\section{Responses to translation during earth-fixed target stabilization}

To address further the degree to which cell responses reflect sensory otolith modulation versus information that has primarily been transformed previously into motor-like signals, we directly compared TrVOR and RVOR responses under earth-fixed target stabilization conditions with those during pursuit of similar ocular amplitude and vergence angle. Thus, peak response amplitude and phase during $0.5 \mathrm{~Hz}$ lateral translation while animals fixated an earth-fixed target have been plotted as a function of cell peak modulation amplitude and phase during $0.5 \mathrm{~Hz}$ horizontal pursuit in Figure $8 A$. Similarly, peak response modulation and phase during head rotation are plotted as a function of peak horizontal
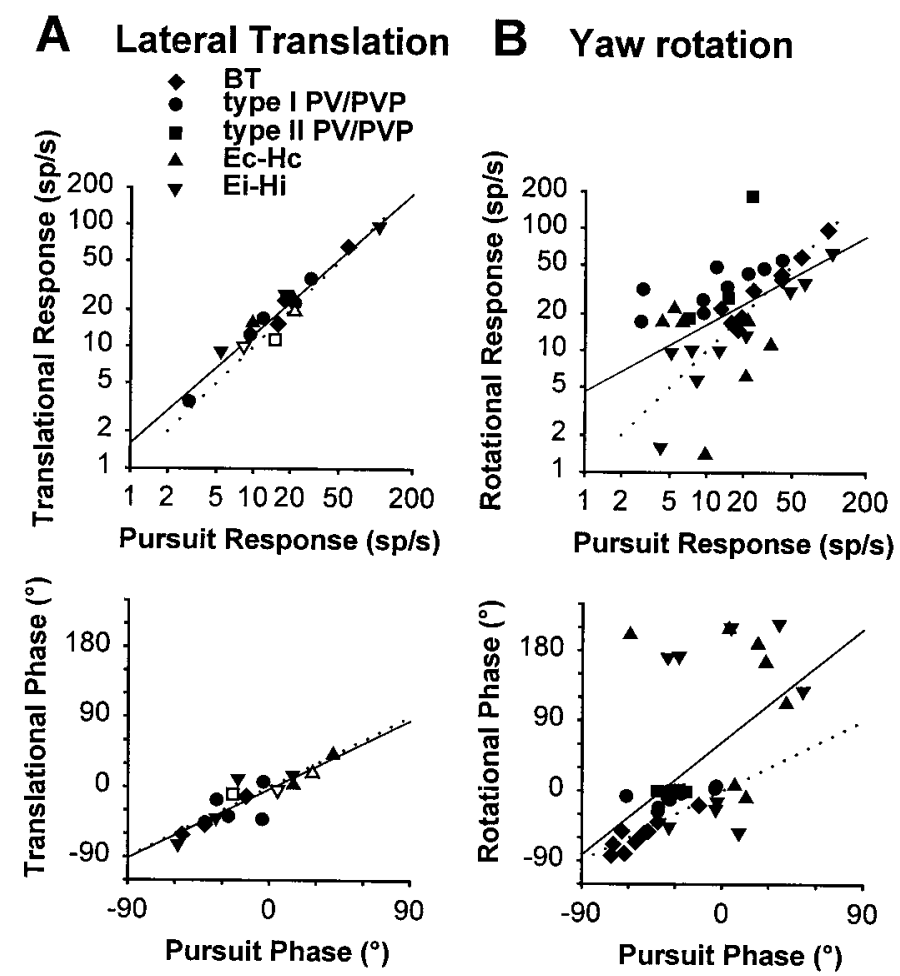

Figure 8. Functional distinction between sensory and motor signals during lateral translation $(A)$ and yaw rotation $(B)$. This is illustrated by plotting the peak firing rate (top) and phase (bottom) during movement while an animal fixated a central earth-fixed target versus the corresponding peak firing rate and phase during horizontal smooth pursuit with the animal stationary. The stimuli during translation and rotation were adjusted such that the elicited eye movements were close to identical to those during smooth pursuit $\left(0.5 \mathrm{~Hz}, \pm 10^{\circ}\right)$. Different symbols are used for different classes of cells (BT, type I PV/PVP, type II PV/PVP, Ec-Hc, and $\mathrm{Ei}-\mathrm{Hi})$. Open symbols in $A$ correspond to the few (3) cells that were tested for both stable gaze and suppression conditions and exhibited small but consistent responses during TrVOR suppression (22-68 spikes $\left.\cdot \mathrm{sec}^{-1} \cdot g^{-1}\right)$. Dotted lines with unity slope indicate equal responses during head movement and pursuit. Solid lines are linear regressions $(A$, top, slope $=0.89, R^{2}=0.94 ; A$, bottom, slope $=0.98, R^{2}=0.77 ; B$, top, slope $=0.55, R^{2}=0.28 ; B$, bottom, slope $\left.=1.61, R^{2}=0.30\right)$. See previous figure legends for abbreviations.

pursuit modulation and phase in Figure $8 B$. To facilitate comparison of the neural responses associated with each stimulus, the parameters of the respective stimuli were chosen such that the evoked eye movements were similar during lateral translation, yaw rotation, and horizontal pursuit (i.e., the same frequency, same vergence angle, and similar peak eye position and velocity; see Materials and Methods). This analysis facilitates a direct comparison of the rotational and translational responses of each cell with those during an equivalent eye movement in the absence of head motion, without assumptions about the signal content and linearity of cell activities.

Under these conditions, neurons with motor-like responses would be expected to respond identically to head-stationary pursuit and lateral translation while an earth-fixed target was stabilized. As seen in Figure $8 A$, this indeed seems to be the case for cell responses during head translation that fall along the unityslope dotted line when plotted as a function of their respective activities during pursuit. This observation holds true even for the few cells that exhibited a consistent response modulation during TrVOR suppression (Fig. 8A, open symbols) and can be explained 
by the fact that, in general, cell modulations during $0.5 \mathrm{~Hz}$ TrVOR suppression were significantly smaller than were their activities under stable gaze conditions $[85 \pm 82$ spikes $\cdot \sec ^{-1} \cdot g^{-1}(n=18)$ vs $616 \pm 677 \mathrm{spikes} \cdot \sec ^{-1} \cdot g^{-1}(n=$ 9); range $\left.211-2366 \mathrm{spikes} \cdot \mathrm{sec}^{-1} \cdot g^{-1}\right)$. It is interesting, however, to contrast this motor-like behavior during translation with the case of head rotation, in which the majority of PV/PVP and eye-head neuron responses demonstrated clear evidence of a sensory canal signal under earth-fixed target stabilization conditions. When peak rotational responses are plotted versus those during pursuit, the data points do not fall along the unity-slope dotted line (Fig. 8B, top). For example, data points for PV/PVP cells fall above the unity-slope dotted line, reflecting the fact that their responses during rotation under stable gaze conditions were larger than were those during an identical target pursuit. Thus, their responses clearly reflect a contribution of sensory signals from the canals during head rotation. The eye-head cell data typically fall below the unity line slope (i.e., smaller amplitude responses during stable gaze than during pursuit) (Fig. 8B, top). In contrast, during translation the activities of all eye movementsensitive cells appear to dominantly reflect the underlying oculomotor behavior.

In Figure 8 neural response phases during head rotation and translation are also plotted against those observed during headstationary target tracking. To facilitate comparison, all neural phases during horizontal pursuit were expressed relative to eye velocity in the interval between -90 and $+90^{\circ}$, independently of the oculomotor preference of the cell (i.e., whether it exhibited an ipsilaterally or contralaterally directed eye movement sensitivity). Phase during head movement was expressed in the range of -90 to $+90^{\circ}$, only if the modulation of a cell relative to eye velocity during the head movement was consistent (i.e., in the same direction) with that during head-stationary pursuit. Otherwise, if a cell demonstrated oppositely directed phases during target tracking and head movement while an earth-fixed target was stabilized (which was often the case for E-H cells; Fig. $8 B$, bottom), its phase was expressed in the range of $90-270^{\circ}$ (i.e., phase was shifted by $180^{\circ}$ ).

Despite a wide distribution of pursuit phases (Scudder and Fuchs, 1992; see also Lisberger et al., 1994), translational response phases closely match those during pursuit (Fig. $8 \mathrm{~A}$, bottom). Thus, all neurons with ipsilaterally directed eye movement sensitivity were excited during contralateral translation, whereas all neurons with contralaterally directed eye movement sensitivity were excited during ipsilateral translation. As described above, this was not in general the case during rotation, particularly for those E-H cells that did not reverse phase during RVOR-stable gaze as compared with RVOR-suppression conditions (Fig. 8B, bottom, plotted in the upper cluster). Thus, a direct comparison of cell responses during $0.5 \mathrm{~Hz}$ translation and pursuit supports the conclusion that cell activities during head translation are dominated by oculomotor-like (or pursuit-related) signals in contrast to the case of head rotation in which the influence of both motor-related and sensory vestibular signals are readily evident in cell responses.

\section{Model structure and simulations}

Several hypotheses have been put forward in the past years to account for the differences in the dynamic processing of sensory signals in the TrVOR as compared with the RVOR (Paige and Tomko, 1991b; Raphan et al., 1996; Telford et al., 1997; Green and Galiana, 1998, 1999; Musallam and Tomlinson, 1999). The goal of this section is two-fold: first, to justify the structure of the model supported here (see Fig. 10) in the context of previous modeling efforts of the VORs; and second, to simulate model cell responses and examine whether the observed differences in the sensitivity and dynamics of eye-ipsi and eye-contra neurons could be predicted by the model structure.

\section{Feedforward and feedback realizations for the RVOR}

To illustrate the conceptual process for the model, let us first consider a classical feedforward realization for the RVOR network (see also Skavenski and Robinson, 1973; Robinson, 1981) that involves a parallel set of pathways from the semicircular canals $[\mathrm{C}(\mathrm{s})]$ to extraocular motoneurons (Fig. 9A). The function of these parallel pathways, consisting of an indirect pathway via a nearly perfect neural integrator (with time constant $>20 \mathrm{sec}$; illustrated for simplicity as $1 / s$, where $s$ is the Laplace operator representing complex frequency) and a direct sensory projection via the vestibular nucleus, is twofold. The first function is to provide a neural integration of sensory signals in the RVOR so that velocity signals originating from the semicircular canals are transformed into eye position commands. Second, the combined effect of the direct and integrator pathways results in a highfrequency lead that cancels the lag associated with the low-pass filter properties of the eye plant $P(s)$. For this cancellation to be effective, the relative weights for the contribution of each pathway are determined by the dynamic characteristics of the eye plant. For example, if $P(s)=1 /\left(1+T_{p} s\right)\left[T_{p} \cong 0.25 \mathrm{sec}\right.$ (Robinson, 1981)] and if the gain of the integrator pathway is assumed to be 1 , the direct pathway must have a gain equal to $T_{p}\left(T_{p}=0.25 \mathrm{sec}\right.$ in Fig. 9A).

An alternative feedback realization of the RVOR was proposed by Galiana and Outerbridge (1984) (outlined in Fig. 9B). The basic structure of the model incorporates a lumped premotor eye movement-sensitive cell type (EM) interconnected in a positive feedback loop with a neural filter, $F(s)$, that represents an internal model of the eye plant $[F(s)=P(s)]$. The output of $F(s)$ provides an internal estimate (efference copy) of eye position, $E^{*}$, that can represent eye position-sensitive neurons in the nucleus prepositus hypoglossi (PH). The input/output properties of this realization are equivalent to the feedforward parallel pathway model of Robinson (1981) (Fig. 9A). However, the feedback realization is more realistic in terms of the highly interconnected nature of the premotor circuitry (cf. McCrea et al., 1981, 1987). Furthermore, the neural integration relies on distributed positive feedback loops around a neural filter with a relatively small time constant (i.e., equal to the dominant eye plant time constant of $\sim 0.25 \mathrm{sec}$ ), as opposed to a localized integrator with a large time constant. In fact, lesion studies have demonstrated that the neural integrative properties of the premotor network involve several brain areas including the vestibular nuclei, the $\mathrm{PH}$, and the cerebellum (Robinson, 1974; Cannon and Robinson, 1987; Cheron and Godaux, 1987; Kaneko, 1997).

\section{Hypotheses for the dynamic processing in the TrVOR pathways} Because primary otolith afferents encode linear head acceleration (as compared with the angular head velocity signals provided by the semicircular canals), the processing described above for semicircular canal signals in the RVOR is not appropriate for the processing of otolith signals in the TrVOR. The most widespread hypothesis for the dynamic processing of otolith signals has been based on a proposal by Paige and colleagues that the additional required low-pass filtering of otolith signals in the TrVOR is 
A

Feedforward Model for the RVOR

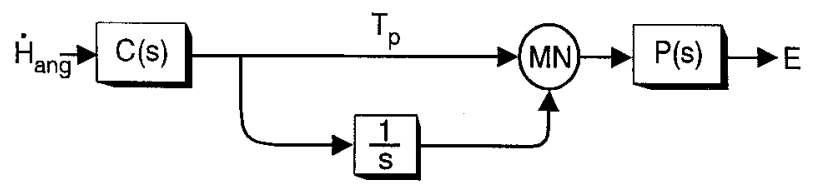

B

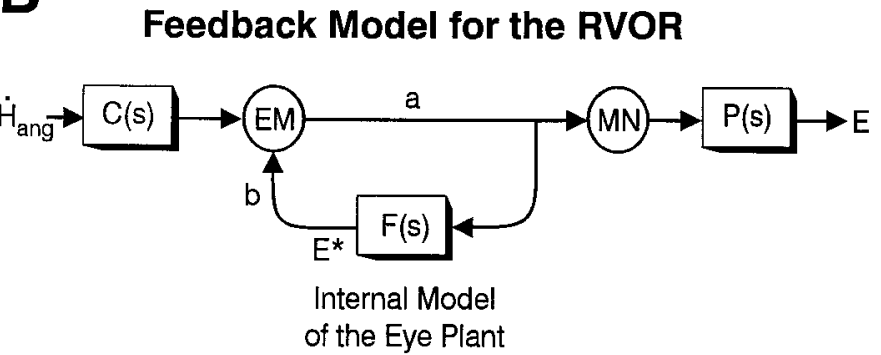

C

Feedforward Model for the RVOR and TrVOR

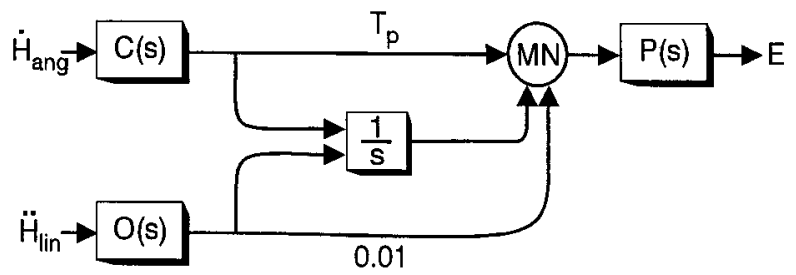

D Feedback Model for the RVOR and TrVOR

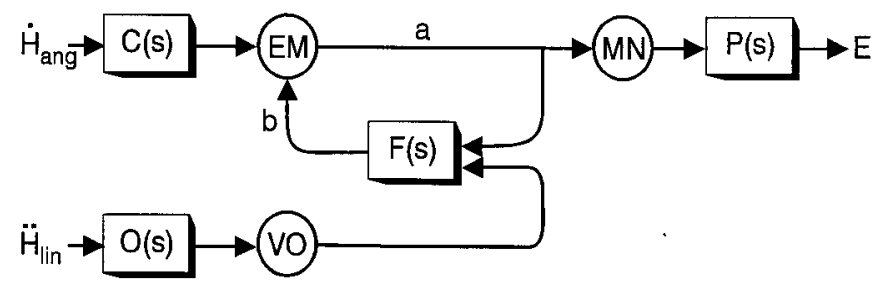

Figure 9. Feedfoward and feedback models of the RVOR $(A, B)$ (Skavenski and Robinson, 1973; Robinson, 1981; Galiana and Outerbridge, 1984) and the RVOR and TrVOR $(C, D)$ [modified following Green and Galiana (1998); Musallam and Tomlinson (1999)]. The neural filter $F(s)$ represents an internal model of the eye plant [i.e., $F(s)=P(s)$ ] that is presumed to exist in the nucleus prepositus hypoglossi $(P H)$. Parameter $T_{p}$ in $A$ and $C$ is chosen to provide neural compensation for the low-pass filter dynamics of the eye plant (Robinson, 1981), whereas parameters $a$ and $b$ in $B$ and $D$ are set to provide an appropriate RVOR gain and integrator time constant (Galiana and Outerbridge, 1984). $C(s)$, Semicircular canals; $E$, eye position; $E^{*}$, efference copy or internal estimate of $E ; E M$, eye movement-sensitive vestibular neurons; $\dot{H}_{\text {ang }}$, angular head velocity; $\ddot{H}_{\text {lin }}$, linear head acceleration; $M N$, extraocular motoneurons; $O(s)$, otolith organs; $V O$, vestibular-only neurons. See previous figure legends for other abbreviations.

performed by a preliminary neural processing stage including either an integrator or a low-pass filter cascaded with a high-pass filter (Paige and Tomko, 1991b; Telford et al., 1997). The prefiltered otolith signals are then combined with the angular head velocity signals from the semicircular canals and are commonly processed via the parallel pathways proposed by Skavenski and Robinson (1973), as outlined above.

An alternative strategy to the "prefiltering" hypothesis has been proposed that we will refer to here as the "eye plant" hypothesis (Green and Galiana, 1998, 1999; Musallam and Tomlinson, 1999). Briefly, the eye plant hypothesis proposes that no additional neural filtering of otolith signals is required if, in contrast to the case of the RVOR, the dynamic characteristics of the eye plant remain uncompensated. The eye plant itself then provides the second required low-pass filtering of otolith signals in the TrVOR (Green and Galiana, 1998). In a Robinson-style feedforward model, the eye plant hypothesis can be realized as illustrated in Figure $9 C$, where the same parallel pathway circuitry is used to process both otolith, $O(s)$, and semicircular canal, $C(s)$, signals. The difference in otolith-ocular versus canal-ocular processing lies in the ratio of the relative projection strengths from each sensor onto the direct and integrator pathways, respectively. This ratio must be equal to the dominant eye plant time constant $T_{p}(\sim 0.25 \mathrm{sec})$ in the case of canal projections to provide compensation for the dominant eye plant pole. However the ratio must be very small in the case of otolith projections [for example, a ratio of $1 / 100$ was used in the simulations of Musallam and Tomlinson (1999); Fig. 9C] so that the resulting lead term in the TrVOR will have a very short time constant, too small to provide a temporal compensation for the dynamics of the eye plant at frequencies below $\sim 16 \mathrm{~Hz}$.
The implementation of the eye plant hypothesis in a positive feedback model of the VOR is illustrated in Figure 9D [according to Green and Galiana (1998)]. Specifically, otolith and canal signals were proposed to join the premotor circuitry at unique sites such that the premotor eye movement-sensitive vestibular neurons EM involved in the disynaptic RVOR pathways receive direct projections from the semicircular canals but only indirect otolith signal inputs via the neural filter $F(s)$ in the PH. Because there are no direct vestibular afferent projections to the $\mathrm{PH}$ (Baker and Berthoz, 1975; Hikosaka et al., 1978) in the original formulation of Green and Galiana (1998), otolith signals were conveyed to the $\mathrm{PH}$ through a VO interneuron. With the exception that no disynaptic (i.e., no direct) otolith-ocular projection was incorporated in the original formulation of the model of Green and Galiana (1998), the dynamic processing performed by the neural network in Figure $9 D$ is equivalent to that in Figure $9 C$.

Both the eye plant (Green and Galiana, 1998) and the prefiltering hypotheses (Paige and Tomko, 1991b; Telford et al., 1997) can qualitatively predict TrVOR dynamics [although an exact match between experimental data and model predictions is problematic because TrVOR dynamics exhibits nonminimum phase behavior when evaluated in an extended frequency range (Angelaki, 1998)]. This is so because the proposed realizations of these alternative hypotheses are practically identical in terms of the overall input-output characterization of the system. For example, in the model of Telford et al. (1997), the low-pass filtering stage is equivalent to a first-order approximation of the dynamic characteristics of the eye plant (i.e., a low-pass filter with a time constant of $0.25 \mathrm{sec}$ ). In addition, the proposed high-pass filtering stage in the Telford et al. (1997) model produces an effect similar to that of a weak direct pathway at high frequencies (e.g., in Fig. 
9 C). What remains unaddressed in the model of Telford et al. (1997) is why such a central low-pass filtering of otolith signals is necessary, because the effects of this prefiltering will be cancelled downstream by the eye plant compensation network, only to be reintroduced again by the actual eye plant.

We believe that the eye plant hypothesis is more realistic for the following reasons. First, the introduction of an additional neural prefiltering stage is redundant and thus less efficient. Second, if such a central filtering existed, one would not expect eye-ipsi and eye-contra cells to exhibit distinct dynamic characteristics during translation. Third, the eye plant hypothesis is more compatible with the differential short-latency neural connections of the otolith-ocular compared with the semicircular canal-ocular systems (see Discussion). For these reasons, in the schematic diagram illustrated in Figure $10 A$ we have extended the feedback realization of the eye plant hypothesis model proposed by Green and Galiana (1998) by explicitly representing an additional group of neurons with ipsilaterally directed eye movement sensitivity.

In Figure $10 A$ (as in Fig. 9D), otolith signals are conveyed only indirectly to eye-contra neurons $\left(\mathrm{EM}_{\mathrm{C}}\right.$; representing a lumped cell type corresponding to type I PV/PVP and Ec-Hc neurons) that represent the main secondary interneurons in the threeneuron-arc RVOR pathway. We suggest that otolith signals join the premotor pathways through eye-ipsi cells $\left(\mathrm{EM}_{\mathrm{I}}\right)$ that represent a lumped population corresponding to type II PV/PVP and Ei-Hi neurons. These cells are assumed to make weak excitatory projections to the ipsilateral abducens (Fig. 10 A, dotted line). However, the dominant output of cell population $\mathrm{EM}_{\mathrm{I}}$ is to the neural filter $F(s)$ in the PH. Although not illustrated in Figure 10, a VO otolith projection to the $\mathrm{PH}$ is also possible (Fig. 9D). In the simulations presented below and as will be further elaborated on in the Discussion, we argue that the model of Figure $10 \mathrm{~A}$ can both account for our experimental observations and provide a rationale for the observed differences in the short-latency neuroanatomical connectivity of otolith-ocular and canal-ocular pathways.

\section{Model predictions}

The predictions of the model illustrated in Figure $10 \mathrm{~A}$ are briefly investigated here to illustrate that the proposed model can replicate the main characteristics of eye-ipsi and eye-contra cells. The model that was actually simulated is illustrated in Figure $10 B$ and represents a more detailed version of that in Figure $10 A$. Specifically, to incorporate motor projections from the $\mathrm{PH}$ (Fig. 10 $A$, dashed line) (McCrea and Baker, 1985; Langer et al., 1986; Escudero and Delgado-Garcia, 1988; Spencer et al., 1989; Escudero et al., 1992; McFarland and Fuchs, 1992), a collateral PH output projection to the $\mathrm{EM}_{\mathrm{I}}$ cell type was included in the model of Figure $10 \mathrm{~B}$ to maintain the appropriate dynamic processing of canal signals in the RVOR. In addition, projections to and from visuomotor areas have been included (for details, see Green and Galiana, 1998, 1999; Green, 2000). These loops are activated by the presence of visual feedback and provide a simple representation of the pursuit system sufficient to explore model predictions under different visual-vestibular stimulus conditions (Fig. 10B, dashed pathways).

Frequency response predictions of the model during TrVOR suppression are illustrated in Figure 11. The model $\mathrm{EM}_{\mathrm{I}}$ cell exhibits gains and phases with respect to head acceleration that are relatively flat and thus sensory-like, whereas the $\mathrm{EM}_{\mathrm{C}}$ cell population exhibits a low-pass-filtered response relative to linear
A
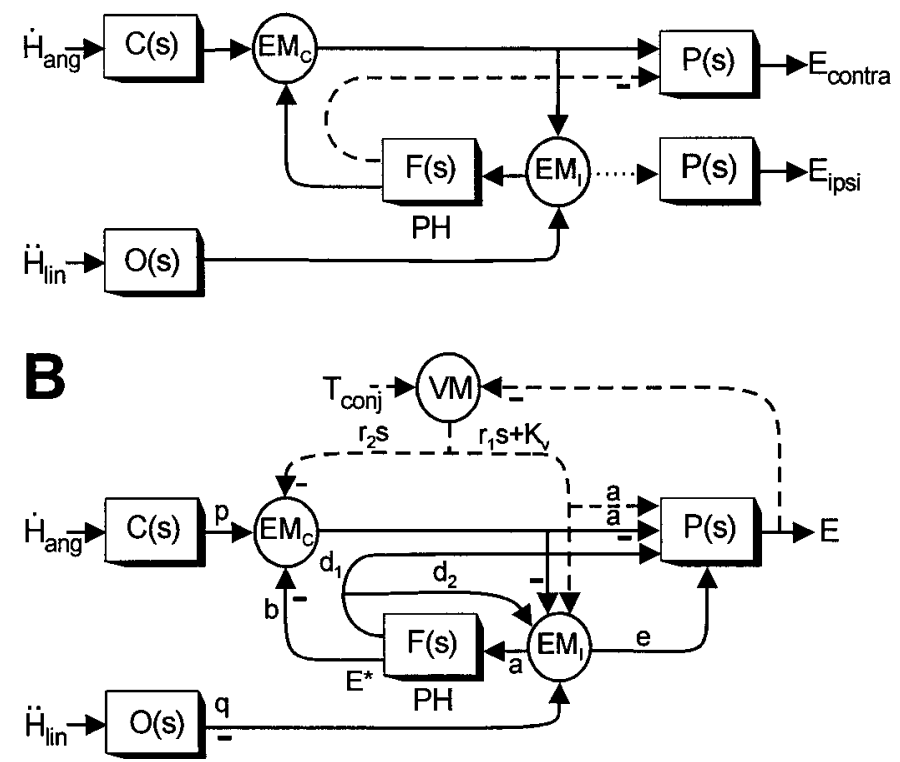

Figure 10. Proposed model of the VORs. $A$, Schematic of the feedback realization of the eye plant hypothesis (Fig. 9D) (Green and Galiana, $1998)$ that has been extended here to include both lumped eye-ipsi $\left(E M_{I}\right)$ and eye-contra $\left(E M_{C}\right)$-sensitive cell types. The dotted line indicates an assumed weak projection from $\mathrm{EM}_{\mathrm{I}}$ cells to the ipsilateral abducens to account for the disynaptic utriculo-ocular pathways (Uchino et al., 1994, 1996). The dashed line indicates an inhibitory projection from $\mathrm{PH}$ neurons [i.e., at the output of $F(s)$ ] to the contralateral abducens (McCrea and Baker, 1985; Langer et al., 1986; Escudero and Delgado-Garcia, 1988) that may account for the inhibitory polysynaptic utriculoabducens pathway (Uchino et al., 1997). $B$, Actual model structure based on the schematic in $A$ that was used to examine the predicted responses of the $\mathrm{EM}_{\mathrm{C}}$ and $\mathrm{EM}_{\mathrm{I}}$ cell types under different visual-vestibular interaction conditions. Dashed pathways associated with visuomotor areas $(V M)$ are activated by the presence of a visual target and represent a simplified lumped pursuit system (Green and Galiana, 1998, 1999; Green, 2000). Negative signs denote projections that on the basis of anatomy are presumed to be either inhibitory to the ipsilateral side of the brain or excitatory to the contralateral side of the brain. Similarly, positive projections (i.e., no negative sign) are assumed to be either excitatory to the ipsilateral side or inhibitory to the contralateral side of the brain. Because the projections in the positive feedback loop interconnecting the $\mathrm{EM}_{\mathrm{C}}$ and $\mathrm{EM}_{\mathrm{I}}$ cells are illustrated as negative (inhibitory), both cell types should be assumed to be located on the same side of the brain in interpreting model simulations. Vestibular stimulation is provided by the angular head velocity $\dot{H}_{\text {ang }}$, sensed by the semicircular canals, $C(s)=T_{c} s /\left(T_{c} s+1\right)$, and linear head acceleration $\ddot{H}_{\text {lin }}$, sensed by the otolith organs, $O(s)=1 /\left(T_{o} s+1\right)$. The negative sign at the output of $O(s)$ indicates that afferents associated with the medial portions of the utricular maculae (excited for contralaterally directed translation) are assumed to provide the TrVOR drive. $T_{c o n j}$ describes the conjugate target position in a head-fixed reference frame. The neural filter, $F(s)=K_{f} /\left(T_{f} s+1\right)$, represents an internal model of the eye plant, $P(s)=K_{p} /\left(T_{p} s+1\right)$, when $T_{f}=T_{p}$. The output of the model, $E$, represents conjugate eye position. Model parameters are as follows: $a=0.19, b=0.75, d_{1}=0.21, d_{2}=1.1, e=0.03, K_{p}=1, K_{f}=2.81, K_{v}=$ 9.51, $p=1, q=0.27, r_{1}=-0.1, r_{2}=0.1, T_{f}=0.25, T_{p}=0.25, T_{c}=5$, and $T_{o}=0.0159$. Note that to simulate appropriate responses for a vergence angle of $6.4^{\circ}$, the primary otolith projection weight $\mathrm{q}$ was adjusted to produce a TrVOR gain of $1.2 \mathrm{~cm} /{ }^{\circ}$ at $4 \mathrm{~Hz}$ (equivalent to a TrVOR gain of $0.32 \mathrm{~cm} \cdot$ degree $^{-1} \cdot \mathrm{MA}^{-1}$ ). MA, Meter-angles, defined as the reciprocal of viewing distance in meters. See previous figure legends for other abbreviations.

acceleration (Fig. 11, dashed vs solid lines, respectively). Thus, in agreement with our experimental observations (Fig. 7), the model predicts that $\mathrm{EM}_{\mathrm{C}}$ and $\mathrm{EM}_{\mathrm{I}}$ cells will exhibit differential dynamic 


\section{TrVOR Suppression}
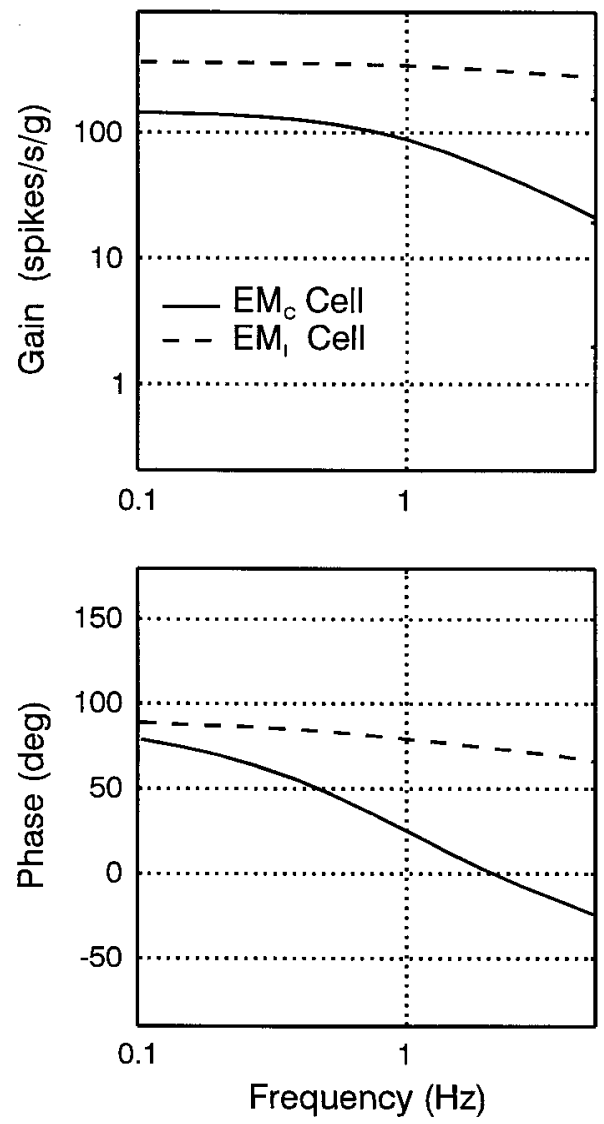

Figure 11. Predicted frequency response plots for the $\mathrm{EM}_{\mathrm{C}}$ and the $\mathrm{EM}_{\mathrm{I}}$ cell types (solid and dashed lines, respectively) in the model of Figure $10 \mathrm{~B}$ during TrVOR suppression. Cell response gains are expressed relative to translational head acceleration. The response phase of the $\mathrm{EM}_{\mathrm{I}}$ cell is expressed relative to contralaterally directed head velocity, whereas the $\mathrm{EM}_{\mathrm{C}}$ cell response phase is expressed relative to ipsilaterally directed head velocity. See previous figure legends for abbreviations.

properties during TrVOR suppression. Analytical descriptions of the model $\mathrm{EM}_{\mathrm{I}}$ and $\mathrm{EM}_{\mathrm{C}}$ cell responses under both head-fixed target stabilization and dark conditions are provided in the Appendix and demonstrate that the model of Figure 10 also predicts different dynamic properties for the two cell populations during translation in darkness. This latter prediction remains to be confirmed experimentally.

Simulated responses of the model $\mathrm{EM}_{\mathrm{C}}$ and $\mathrm{EM}_{\mathrm{I}}$ cell types during smooth pursuit are compared with those for earth-fixed target stabilization during rotation and translation in Figure 12. To duplicate our experimental protocols, stimuli for these simulations were adjusted to generate a similar oculomotor response ( $\pm 10^{\circ}$ sinusoidal ocular deviations). In agreement with Figure 8, model $\mathrm{EM}_{\mathrm{C}}$ cell responses are predicted to be similar during translation and head-stationary pursuit. During head rotation, however, the model $\mathrm{EM}_{\mathrm{C}}$ cell exhibits larger modulations with phases that are more closely aligned with head/eye velocity. Hence, although $\mathrm{EM}_{\mathrm{C}}$ cells are predicted to exhibit motor-like responses during head translation, they clearly reflect the contribution of a sensory canal signal during head rotation, as observed experimentally (Fig. 8, circles, triangles). EM $\mathrm{I}_{\mathrm{I}}$ cells in the model, on the other hand, exhibit comparable gains and phases under all conditions. Notably this was also true for many of the recorded Ei-Hi cells (Fig. 8, inverted triangles).

In summary, despite its limited structure (i.e., lumped cell types and unilateral representation), the predictions of the model in Figure 10 appear to be at least qualitatively consistent with our general experimental observations under both head-fixed and earth-fixed target stabilization conditions. Hence, we believe that our current investigation provides support for the recent proposal that the dynamic processing of sensory signals in the RVOR and TrVOR is achieved by virtue of unique projection sites for canal and otolith signals onto a shared premotor network (Green and Galiana, 1998).

\section{DISCUSSION}

The responses of eye movement-sensitive cell types in the rostral vestibular nuclei that represent important premotor neurons in the RVOR were examined during head translation with the goal of elucidating the role that these cells play in the TrVOR. The results are in agreement with previous eccentric rotation studies (McConville et al., 1996; Chen-Huang and McCrea, 1999) but present new observations that have important implications for the organization of the TrVOR. Specifically, several distinctions in the activities of individual cell types were identified when their rotational and translational responses were directly compared for different visual-vestibular stimulus conditions. First, all eye movement-sensitive vestibular neurons demonstrated modulations during translation that were closely associated with motor responses. This is in contrast to the case of head rotation in which both motor-related and sensory signals are clearly evident in the majority of eye movement-sensitive cell activities. Second, during TrVOR suppression, type I PV/PVP and BT neurons could be distinguished from type II PV/PVP cells and a subpopulation of $\mathrm{E}-\mathrm{H}$ cells in that only the latter cell types demonstrated significant modulations in activity. Notably, the type I PVP cell, traditionally considered the main interneuron in the shortest latency RVOR pathways, displayed no clear evidence of a sensory otolith signal modulation during translation. Third, among the cells that responded during TrVOR suppression, Ec-Hc neurons displayed dynamic characteristics distinct from those sensitive to ipsilaterally directed eye movements (i.e., Ei-Hi and type II PV/PVP cells). Specifically, Ec-Hc neuron responses during TrVOR suppression were lower in sensitivity and reflected a low-pass filtering of the sensory acceleration signal. In contrast, type II PV/PVP and Ei-Hi cells exhibited responses with relatively flat gain characteristics and phases between linear acceleration and velocity.

In the following sections we will attempt to interpret these results in terms of both the current understanding of the neuroanatomy associated with otolith-ocular versus canal-ocular pathways and the different dynamic processing requirements for the TrVOR as compared with the RVOR.

\section{Relationship between specific cell groups and utriculoabducens connectivity}

The classical three-neuron-arc pathways for the horizontal RVOR involve secondary neurons in the vestibular nuclei that receive direct primary afferent inputs from the semicircular canals and make excitatory projections to contralateral abducens motoneurons (Richter and Precht, 1968; Baker et al., 1969; Precht et al., 1969; Schwindt et al., 1973). In contrast, the shortest latency excitatory utriculoabducens pathway is a weak ipsilateral projection, whereas the contralateral projections are at least trisynaptic and inhibitory (Schwindt et al., 1973; Uchino et al., 1994, 1996, 


\section{A Smooth Pursuit}
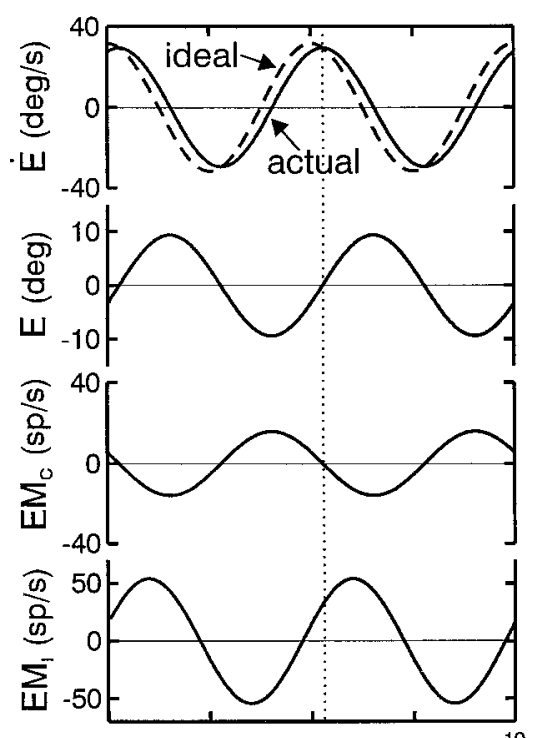

Translation

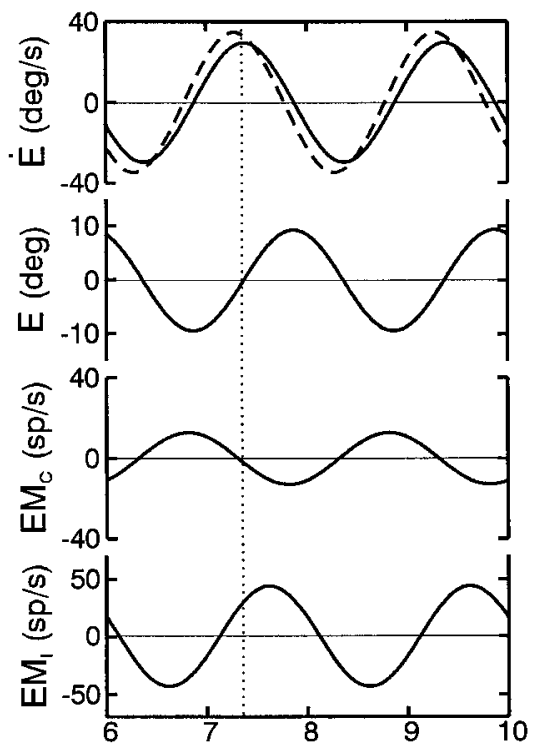

Rotation

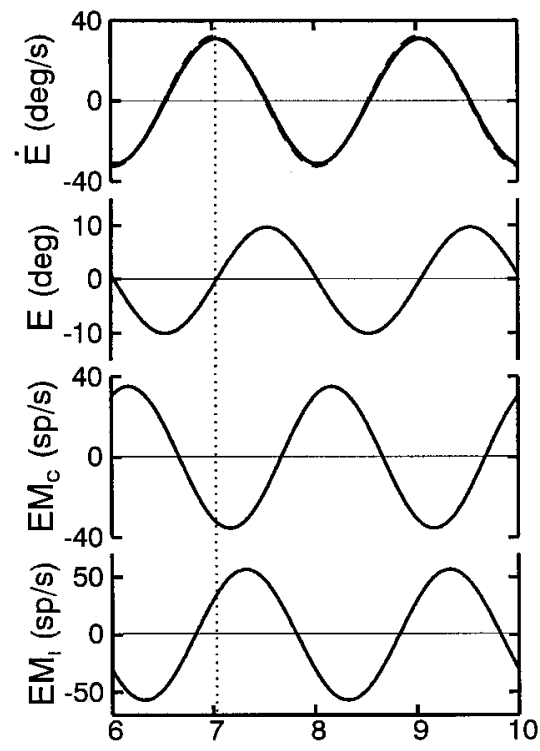

Figure 12. Simulated $\mathrm{EM}_{\mathrm{C}}$ and $\mathrm{EM}_{\mathrm{I}}$ cell responses in the model of Figure $10 B$ for $0.5 \mathrm{~Hz}$ pursuit $(A)$, earth-fixed target stabilization during head translation $(B)$, and earth-fixed target stabilization during head rotation $(C)$. Target or head movement stimuli were adjusted in all cases to elicit ocular deviations of $\pm 10^{\circ}$ for a vergence angle of $6.4^{\circ}$ (as in our experimental conditions). Assuming the convention that leftward ocular deviations are positive, the model $\mathrm{EM}_{\mathrm{C}}$ and $\mathrm{EM}_{\mathrm{I}}$ cell populations are both presumed to be located in the left vestibular nucleus. Hence, $\mathrm{EM}_{\mathrm{C}}$ cells are excited for contralaterally directed eye movements, whereas $\mathrm{EM}_{\mathrm{I}}$ cells are excited for ipsilaterally directed ocular deviations. See previous figure legends for abbreviations.

1997; Imagawa et al., 1995). Because it is known that the large majority of type I PVP neurons make direct excitatory connections to the contralateral abducens (McCrea et al., 1987; Scudder and Fuchs, 1992), one must conclude that primary otolith afferents do not make monosynaptic projections to type I PVP neurons (Fig. 13). In support of this conclusion, none of the type I $\mathrm{PV} / \mathrm{PVP}$ cells in the present study responded during TrVOR suppression, nor did they display differences in their responses during head-stationary pursuit versus head translation while an earth-fixed target was viewed. Similarly, a sensory otolith signal was not obvious in the response properties of BT neurons.

In contrast to the exclusively motor-like signals apparent in type I PV/PVP and BT activities during translation, many type II $\mathrm{PV} / \mathrm{PVP}$ and $\mathrm{E}-\mathrm{H}$ neurons may receive more direct sensory otolith inputs (Fig. 13). Three results support this conclusion. First, a subset of type II PV/PVP and E-H cells modulated during TrVOR suppression in the absence of eye movements. Second, the responses of all type II PV/PVP and Ei-Hi cells during TrVOR suppression were similar in terms of both amplitude and phase to those described previously for the majority of central vestibular-only otolith neurons (Angelaki and Dickman, 2000), suggesting that they most likely represent sensory otolith signals. Finally, the existence of direct otolith inputs onto these cells is consistent with the ipsilateral utriculoabducens pathway (Uchino et al., 1994). In particular, on the basis of their eye movement sensitivities, type II PV/PVP and Ei-Hi cells would drive appropriately directed eye movements if they were excitatory to the ipsilateral abducens nucleus (Fig. 13, question marks) (McCrea et al., 1987). Although such ipsilateral motor projections from these cell types have not been confirmed, we suggest that at least a subset of type II PV/PVP and/or Ei-Hi cells could represent the secondary interneurons in the weak ipsilateral disynaptic utriculoabducens pathway.

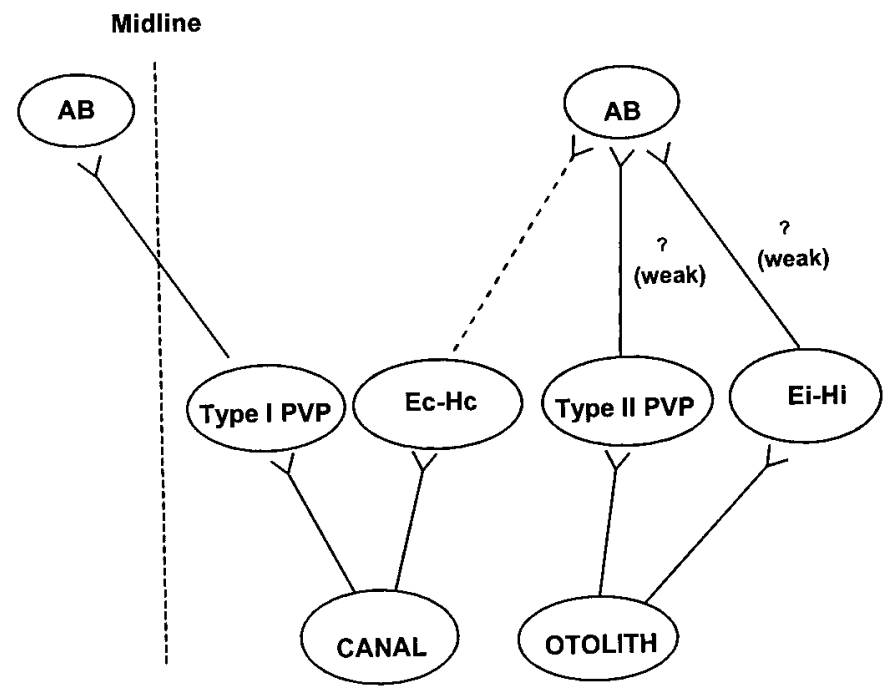

Figure 13. Postulated relationship between specific cell groups and disynaptic utriculoabducens connectivity. The vertical dashed line represents the midline. The dashed line from Ec-Hc cells to the ipsilateral AB represents an inhibitory projection. The question marks illustrate postulated but undocumented weak excitatory projections from the type II $\mathrm{PV} / \mathrm{PVP}$ and Ei-Hi neurons to the ipsilateral abducens nucleus. Polysynaptic connectivities are not included. $A B$, Abducens nucleus. See previous figure legends for other abbreviations.

There are reasons to propose that Ec-Hc cells occupy a distinct location in the premotor pathways associated with sensorimotor transformations in the TrVOR, as compared with cells that code for ipsilaterally directed eye movements (i.e., Ei-Hi and type II PV/PVP cells). First, the response sensitivities of Ec-Hc cells during TrVOR suppression were smaller than were those of eye-ipsi cells. Second, eye-contra and eye-ipsi cells exhibited 
different dynamic properties during translation. Specifically, the Ec-Hc neurons that modulated during TrVOR suppression exhibited sensitivities relative to linear acceleration that decreased with frequency, suggesting a low-pass filtering of otolith signals. In contrast, eye-ipsi neuron dynamics was relatively flat over the frequency range of $0.16-2 \mathrm{~Hz}$ and was similar to that of the majority of rostrally located VO neurons (Angelaki and Dickman, 2000). These observations and the fact that Ec-Hc cells make inhibitory connections to the ipsilateral abducens nucleus (Scudder and Fuchs, 1992), whereas to date only disynaptic excitatory utricular projections to the ipsilateral abducens have been identified, suggest that Ec-Hc cells probably receive otolith signals only indirectly (i.e., not monosynaptically; Fig. 13).

\section{Differences in neuroanatomical topology and processing between TrVOR and RVOR}

These conclusions have been summarized in a simple model for the generation of the VORs (Fig. 10A). The basic structure of the model is based on a recent proposal by Green and Galiana (1998) that illustrated that appropriate RVOR and TrVOR responses may be generated if canal and otolith signals project differentially onto a shared premotor network. The structures in Figure 10 represent one realization of the hypothesis that the low-pass filter dynamics of the eye plant rather than central filtering provides the additional high-frequency temporal integration that is required in the TrVOR as compared with the RVOR pathways (Green and Galiana, 1998, 1999; Musallam and Tomlinson, 1999). In the proposed model structure, otolith signals are conveyed directly to $\mathrm{EM}_{\mathrm{I}}$ cells but only indirectly to $\mathrm{EM}_{\mathrm{C}}$ cells via the neural filter $F(s)$ that is shared with the RVOR pathway. As a result, otolith information appears differentially processed relative to the sensory signal on each cell type (see simulations in Fig. 11 ), in agreement with our experimental observations during TrVOR suppression (Fig. 7). Model cell predictions are also consistent with the experimental observation that during $0.5 \mathrm{~Hz}$ translation while an earth-fixed target was stabilized, all eye movement-sensitive cell types exhibited responses that were comparable with those during head-stationary pursuit. Although our current study focused entirely on cell responses during translation under visual-vestibular interaction conditions, a key model prediction is that $\mathrm{EM}_{\mathrm{C}}$ and $\mathrm{EM}_{\mathrm{I}}$ cells will also demonstrate differential dynamic properties in the dark. Hence, an investigation of eye movement-sensitive cell activities in the absence of visual feedback will be important in confirming the viability of the proposed structure.

Of particular relevance is the fact that the model of Figure 10 is not only compatible with, but also provides a functional explanation for, the known differences in the neuroanatomy of otolithocular versus canal-ocular pathways. Specifically, the projections from $\mathrm{PH}$ neurons [output of $F(s)$ ] to the contralateral abducens in the model can account for the polysynaptic contralateral inhibitory utriculoabducens projections (Uchino et al., 1994, 1996). The model also remains consistent with the disynaptic ipsilateral utriculoabducens connectivity, if $\mathrm{EM}_{\mathrm{I}}$ cells make weak excitatory projections to the ipsilateral abducens (Fig. 10A,dotted line). The dominant projection of cell $\mathrm{EM}_{\mathrm{I}}$ is, however, proposed to be to the $\mathrm{PH}$ so that otolith signals are conveyed to motor neurons mainly indirectly via neural filter $F(s)$ that is shared with the semicircular canal pathway.

It is important to point out that although the shortest latency utriculo-ocular pathway is ipsilateral and does not involve the type I PVP neurons, this does not by any means imply that the type I PVPs do not contribute to the TrVOR (see also ChenHuang and McCrea, 1999). Type I PVPs and the neurons in the $\mathrm{PH}$ represent the main oculomotor projections to the abducens nuclei, as compared with the much weaker disynaptic ipsilateral utriculoabducens connections. Thus, in contrast to the case of the RVOR in which the disynaptic three-neuron-arc pathway is a heavy projection, the bulk of the signals to move the eyes during translation seem to use polysynaptic (minimum trisynaptic) pathways. Such pathways could still elicit eye movements at short latency, $\sim 8-12 \mathrm{msec}$, as has been shown for the TrVOR (Angelaki and McHenry, 1999).

It should be added that, although the model of Figure 10 does not incorporate an involvement of the cerebellar flocculus/ventral paraflocculus (FL/VPF), some of the postulated eye-ipsi and eye-contra neurons might be flocculus target neurons (FTNs) (Lisberger and Pavelko, 1988; Lisberger et al., 1994; Zhang et al., 1995). In fact, a FL/VPF projection might account for the response reversal of many E-H cells during translation under stable gaze and suppression conditions. Because the main conclusions of the present study are independent of these projections and because we did not identify our neurons as FTNs, we have not explicitly incorporated the role of the FL/VPF in Figure 10. The model of Figure 10 also does not address other aspects of the TrVOR properties, such as the dependence on viewing distance and gaze direction as well as the role of the bilateral labyrinths and different otolith afferent contributions (Schwarz et al., 1989; Paige and Tomko, 1991a,b; Schwarz and Miles, 1991; Telford et al., 1997; Angelaki et al., 2000b,c; McHenry and Angelaki, 2000; Angelaki and Hess, 2001). A more detailed investigation of these topics requires further studies.

In summary, our current results demonstrate that the eyecontraversive cell types involved in the shortest latency RVOR pathways either exhibit negligible responses during TrVOR suppression (i.e., type I PV/PVP and approximately one-third of Ec-Hc cells) or responses that reflect low-pass-filtered linear acceleration (i.e., the majority of Ec-Hc cells), suggesting that these cell types receive mainly indirect sensory otolith signals. In contrast, eye-ipsiversive cell types (the majority of type II PV/ PVP and approximately one-half of Ei-Hi cells) exhibited on average a more robust modulation during TrVOR suppression, with dynamics that would be consistent with otolith sensory signals. Thus, our results provide evidence of a differential processing of sensory information on $\mathrm{EM}_{\mathrm{C}}$ versus $\mathrm{EM}_{\mathrm{I}}$ cell types. Our current neurophysiological observations as well as previous neuroanatomical data are consistent with a model for the RVOR and TrVOR pathways that postulates differential projections of canal and otolith signals onto $\mathrm{EM}_{\mathrm{C}}$ and $\mathrm{EM}_{\mathrm{I}}$ cells, respectively. Furthermore, although recent studies have focused on the responses of premotor $\mathrm{EM}_{\mathrm{C}}$ cells involved in the disynaptic RVOR pathways, here we suggest a prominent role for $\mathrm{EM}_{\mathrm{I}}$ cells in the TrVOR. The role of these cells is nevertheless less important in terms of their direct oculomotor projections than in terms of their function as a conduit for otolith signals in polysynaptic TrVOR pathways, required to ensure that canal and otolith signals project onto a shared network at unique sites so that sensory vestibular signals with distinct dynamic properties may be differentially processed by a common premotor network.

\section{APPENDIX}

We have summarized here analytical descriptions of the responses of the lumped $\mathrm{EM}_{\mathrm{C}}$ and the $\mathrm{EM}_{\mathrm{I}}$ cell types in the model of Figure $10 \mathrm{~B}$ under both dark and TrVOR suppression condi- 
tions. To illustrate the model predictions, Laplace domain descriptions of the responses of each cell type were derived from the model in Figure $10 \mathrm{~B}$ by treating the schematic as a signal flow graph in which each model parameter represents the gain associated with a given pathway, circles (e.g., $\mathrm{EM}_{\mathrm{C}}$ and $\mathrm{EM}_{\mathrm{I}}$ cells) represent simple summing junctions and boxes [e.g., $F(s)$ and $P(s)$ ] represent dynamic elements or filters. A summing junction is assumed at the input of each box such that all inputs to the filter are summed before the filtering process is applied. Because the goal of the modeling investigation was not to replicate detailed dynamic characteristics of behavioral and cell responses but rather to illustrate that the general predictions of the model structure are qualitatively consistent with our experimental observations, the following simplified representations of the sensors and eye plant were chosen. (1) A first-order low-pass model was used to represent the eye plant dynamics: that is, $P(s)=K_{p} /\left(T_{p} s+1\right)$, where $T_{p}=0.25$ sec (Robinson, 1981). Similarly, the neural filter $F(s)$ represents an internal model of the eye plant such that $F(s)=K_{f}\left(T_{f} s+1\right)$, where $T_{p}=T_{f}=T$ (Galiana and Outerbridge, 1984). (2) Canal afferent dynamics relative to head velocity is approximated by a first-order high-pass filter: $C(s)=T_{c} s /\left(T_{c} s+1\right)$ (Fernández and Goldberg, 1971). (3) Otolith afferent dynamics relative to head acceleration was modeled as a first-order system with a pole at $10 \mathrm{~Hz}: O(s)=$ $1 /\left(T_{o} s+1\right)$ (Fernández and Goldberg, 1976c).

Model predictions regarding the sensitivity and phase of the RVOR and TrVOR, as well as those of the $\mathrm{EM}_{\mathrm{C}}$ and $\mathrm{PH}$ cell types [output of $F(s)$ ] during rotation and translation, have been reported previously (Green and Galiana, 1998, 1999; Green, 2000). Here we focus on Laplace domain descriptions of $\mathrm{EM}_{\mathrm{I}}$ and $\mathrm{EM}_{\mathrm{C}}$ cell responses to canal and otolith stimulation both in darkness and under VOR suppression conditions. Because the rotational responses of eye movement-sensitive neurons in a feedback realization of the RVOR have been described previously (Galiana and Outerbridge, 1984; Green, 2000), discussion of the analytical expressions for the model $\mathrm{EM}_{\mathrm{C}}$ and $\mathrm{EM}_{\mathrm{I}}$ cells will focus on the component of their activities related to otolith stimulation during head translation.

\section{Dark conditions}

Under dark conditions the following set of equations may be written to describe the model system:

$$
\begin{gathered}
\operatorname{EM}_{\mathrm{C}}(s)=\mathrm{pC}(s) \dot{H}_{\text {ang }}(s)-b E^{*}(s), \\
\operatorname{EM}_{\mathrm{I}}(s)=-q O(s) \ddot{H}_{\text {lin }}(s)-\operatorname{EM}_{\mathrm{C}}(s)+d_{2} E^{*}(s), \\
E^{*}(s)=a \operatorname{EM}_{\mathrm{I}}(s) F(s), \\
E(s)=-a \operatorname{EM}_{\mathrm{C}}(s) P(s)+d_{1} E^{*}(s) P(s)+e \operatorname{EM}_{\mathrm{I}}(s) P(s) .
\end{gathered}
$$

Solution of the above system equations yields the following expressions describing $\mathrm{EM}_{\mathrm{C}}$ and $\mathrm{EM}_{\mathrm{I}}$ cell responses to canal and otolith stimulation:

$$
\begin{gathered}
\operatorname{EM}_{\mathrm{C}}(s)=\frac{p G_{\mathrm{cVCD}} G_{D}\left(T_{\mathrm{cVCD}} s+1\right)}{\left(T_{O} s+1\right)} \frac{T_{c} s}{\left(T_{c} s+1\right)} \dot{H}_{\mathrm{ang}}(s) \\
+\frac{q G_{\mathrm{oVCD}} G_{D}}{\left(T_{D} s+1\right)} \frac{1}{\left(T_{O} s+1\right)} \ddot{H}_{\mathrm{lin}}(s), \\
\operatorname{EM}_{\mathrm{I}}(s)=-\frac{p G_{\mathrm{cVID}} G_{D}\left(T_{\mathrm{cVID}} s+1\right)}{\left(T_{D} s+1\right)} \frac{T_{c} s}{\left(T_{c} s+1\right)} \dot{H}_{\mathrm{ang}}(s)
\end{gathered}
$$

$$
-\frac{q G_{\mathrm{oVID}} G_{D}\left(T_{\mathrm{oVID}} s+1\right)}{\left(T_{D} s+1\right)} \frac{1}{\left(T_{O} s+1\right)} \ddot{H}_{\mathrm{lin}}(s),
$$

where dark system gain terms $G_{\mathrm{xxD}}$ and time constants $T_{\mathrm{xxD}}$ are expressed in terms of model parameters as:

$$
\begin{gathered}
G_{\mathrm{cVCD}}=\left(1-d_{2} a K_{f}\right), \quad T_{\mathrm{cVCD}}=T /\left(1-d_{2} a K_{f}\right), \\
G_{\mathrm{oVCD}}=a K_{f} b, \quad G_{\mathrm{cVID}}=1, \quad T_{\mathrm{cVID}}=T, \\
G_{\mathrm{oVID}}=1, \quad T_{\mathrm{oVID}}=T, \quad G_{D}=1 /\left(1-a b K_{f}-d_{2} a K_{f}\right), \\
\text { and } \quad T_{D}=T /\left(1-a b K_{f}-d_{2} a K_{f}\right) .
\end{gathered}
$$

Under dark conditions, the system is characterized by a pole associated with the time constant $T_{D}$. Model parameters (see Fig. 10 legend) were adjusted to set $T_{D}$ to a large value of $\sim 20 \mathrm{sec}$ such that the system provides an integration of vestibular signals at frequencies above $\sim 0.01 \mathrm{~Hz}$ (i.e., this pole represents the central neural integrator). During head translation, the response of the $\mathrm{EM}_{\mathrm{C}}$ cell reflects this pole, and hence otolith signals on this cell type appear to have been integrated relative to linear acceleration (Green and Galiana, 1998, 1999). The expression for the EMI cell, in addition to being characterized by a pole associated with the neural integrator time constant $T_{D}$, also includes a zero (lead term) associated with the time constant $T_{\text {oVID }}=T=0.25 \mathrm{sec}$ [notice that the expression for $\operatorname{EM}_{\mathrm{C}}(s)$ is not characterized by such a zero]. As a result, although the $\mathrm{EM}_{\mathrm{I}}$ cell is expected to modulate in-phase with linear head velocity at low frequencies, at frequencies above $\sim 0.6 \mathrm{~Hz}$ the zero cancels the effects of the pole. During high-frequency translation in the dark, $\mathrm{EM}_{\mathrm{I}}$ cells are therefore predicted to exhibit responses that code more closely for linear head acceleration, whereas $\mathrm{EM}_{\mathrm{C}}$ cells are predicted at these same high frequencies to exhibit responses closely associated with linear head velocity.

\section{Head-fixed target-viewing conditions}

Under visual feedback conditions, the dashed pathways in the schematic of Figure $10 B$ are active, and the model system is now described by the equations:

$$
\begin{aligned}
& \operatorname{EM}_{\mathrm{C}}(s)=p C(s) \dot{H}_{\text {ang }}(s)-b E^{*}(s)-r_{2} s\left(T_{\text {conj }}(s)-E(s)\right), \\
& \operatorname{EM}_{\mathrm{I}}(s)=-q O(s) \ddot{H}_{\text {lin }}(s)-\operatorname{EM}_{\mathrm{C}}(s)+d_{2} E^{*}(s) \\
& +\left(r_{1} s+K_{v}\right)\left(T_{\text {conj }}(s)-E(s)\right), \\
& E^{*}(s)=a \operatorname{EM}_{\mathrm{I}}(s) F(s), \\
& E(s)=-a \operatorname{EM}_{\mathrm{C}}(s) P(s)+d_{1} E^{*}(s) P(s)+e \mathrm{EM}_{\mathrm{I}}(s) P(s) \\
& +a\left(r_{1} s+K_{v}\right)\left(T_{\text {conj }}(s)-E(s)\right) .
\end{aligned}
$$

Under head-fixed target-viewing conditions, there is no movement of the target relative to the head (i.e., the target and head move in tandem). Therefore, assuming a centrally located target such that $T_{\text {conj }}=0$, the terms associated with $T_{\text {conj }}$ drop out of the equations. The equations are further simplified by imposing the condition $d_{1}=d_{2} a$ [i.e., the strength of $\mathrm{PH}$ output projections to $P(s)$ and $F(s)$ are equal] that is required in the model to ensure that RVOR dynamics is appropriate and that the output of $F(s)$ provides an accurate internal estimate of eye position $E^{*}$ during head rotation and smooth pursuit. After incorporation of these simplifications, the following expressions may be written to de- 
scribe $\mathrm{EM}_{\mathrm{C}}$ and $\mathrm{EM}_{\mathrm{I}}$ cell responses under head-fixed targetviewing conditions:

$$
\begin{aligned}
& \operatorname{EM}_{\mathrm{C}}(s)=\frac{p G_{\mathrm{cVCL}} G_{L}\left(T_{\mathrm{cVCL}} s+1\right)}{\left(T_{L} s+1\right)} \frac{T_{c} s}{\left(T_{c} s+1\right)} \dot{H}_{\mathrm{ang}}(s) \\
& +\frac{q G_{\mathrm{oVCL}} G_{L}\left(T_{\mathrm{oVCL} 1} s+1\right)\left(T_{\mathrm{oVCL} 2} s+1\right)}{\left(T_{L} s+1\right)} \frac{1}{(T s+1)} \frac{1}{\left(T_{o} s+1\right)} \ddot{H}_{\mathrm{lin}}(s), \\
& \mathrm{EM}_{1}(s)=-\frac{p G_{\mathrm{cVIL}} G_{L}\left(T_{\mathrm{cVIL}} s+1\right)}{\left(T_{L} s+1\right)} \frac{T_{c} s}{\left(T_{c} s+1\right)} \dot{H}_{\mathrm{ang}}(s)
\end{aligned}
$$

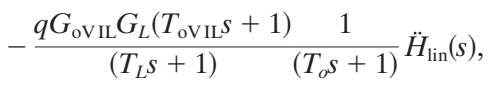

where gains $G_{\mathrm{xxL}}$ and time constants $T_{\mathrm{xxL}}$ under light conditions are expressed in terms of model parameters as:

$$
\begin{gathered}
G_{\mathrm{cVCL}}=\left(1-d_{2} a K_{f}+K_{v} K_{p}(a+e)\right) \\
T_{\mathrm{cVCL}}=\left(T+r_{1} K_{p}(a+e)\right) /\left(1-d_{2} a K_{f}+K_{v} K_{p}(a+e)\right), \\
G_{\mathrm{cVIL}}=1, \quad T_{\mathrm{cVIL}}=T, \\
G_{\mathrm{oVIL}}=\left(1+K_{v} K_{p} a\right), \quad T_{\mathrm{oVIL}}=\left(T+\left(r_{1}+r_{2}\right) a K_{p}\right) /\left(1+K_{v} K_{p} a\right), \\
G_{L}=1 /\left(1-a b K_{f}-d_{2} a K_{f}+K_{v} K_{p}(a+e)\right),
\end{gathered}
$$

and $T_{L}=\left(T+\left(r_{1}+r_{2}\right)(a+e) K_{p}\right) /\left(1-a b K_{f}-d_{2} a K_{f}+K_{v} K_{p}(a\right.$

Time constants $T_{\mathrm{oVCL} 1}$ and $T_{\mathrm{oVCL} 2}$ and gain $G_{\mathrm{oVCL}}$ are obtained from the expressions:

$$
\begin{aligned}
& T_{\mathrm{oVCL} 1}=-\frac{2 a^{\prime}}{-b^{\prime}+\sqrt{b^{\prime 2}-4 a^{\prime} c^{\prime}}}, \\
& T_{\mathrm{oVCL} 2}=-\frac{2 a^{\prime}}{-b^{\prime}-\sqrt{b^{\prime 2}-4 a^{\prime} c^{\prime}}}, \\
& \text { and } G_{\mathrm{oVCL}}=-e K_{p} r_{2} T\left(\frac{1}{T_{\mathrm{oVCL} 1}}\right)\left(\frac{1}{T_{\mathrm{oVCL} 2}}\right),
\end{aligned}
$$

where $a^{\prime}, b^{\prime}$, and $c^{\prime}$ are written in terms of model parameters as:

$$
\begin{array}{r}
a^{\prime}=-e K_{p} r_{2} T, \quad b^{\prime}=-a^{2} d_{2} K_{f} K_{p} r_{2}-e K_{p} r_{2}+a K_{f} b\left(T+r_{1} a K_{p}\right), \\
\text { and } c^{\prime}=a K_{f} b\left(1+a K_{v} K_{p}\right) .
\end{array}
$$

The above equations illustrate that similar, but slightly more complex, equations also predict a differential processing of otolith signals on $\mathrm{EM}_{\mathrm{C}}$ and $\mathrm{EM}_{\mathrm{I}}$ cells during TrVOR suppression. Under visual feedback conditions, cell responses are characterized by the system time constant $T_{L}$ that has a small value of $0.12 \mathrm{sec}$, reflecting the model pursuit bandwidth of $\sim 1.3 \mathrm{~Hz}$ (Lisberger et al., 1987; Barnes, 1993). EM I cell responses are again characterized by a lead term associated with a time constant under light conditions of $T_{\text {oVIL }}=0.089 \mathrm{sec}$ (zero at $\sim 1.8 \mathrm{~Hz}$ ). Because this zero and the light system pole are closely matched in frequency, the effect of one cancels that of the other so that the $E_{I}$ cell modulates with sensory-like dynamics at all frequencies (see Fig. 11 , dashed lines). In contrast, the response of the $\mathrm{EM}_{\mathrm{C}}$ cell is characterized by two zeros associated with time constants $T_{\text {ovCL1 }}$ $=0.078 \mathrm{sec}$ and $T_{\mathrm{oVCL} 2}=-0.0085 \mathrm{sec}$ (a negative or left-hand plane zero at $\sim 2 \mathrm{~Hz}$ and a positive or right-hand plane zero at $\sim 19 \mathrm{~Hz}$ ) as well as an additional pole with time constant $T=T_{f}$
$=T_{p}=0.25 \mathrm{sec}(\sim 0.6 \mathrm{~Hz})$. As a result, because the system pole at $1.3 \mathrm{~Hz}$ and the zero at $2 \mathrm{~Hz}$ are relatively closely matched, the effect of one primarily cancels that of the other. However, the additional pole associated with the time constant $T=0.25 \mathrm{sec}$ that appears selectively in the expression for $\operatorname{EM}_{\mathrm{C}}(s)$ gives rise to a falloff in gain and an accompanying phase lag relative to linear acceleration; thus, at frequencies above $\sim 0.6 \mathrm{~Hz}$ the response of the $\mathrm{EM}_{\mathrm{C}}$ cell appears low-pass filtered relative to the sensory otolith signal (Fig, 11, solid lines). The positive (right-hand plane) zero only has an effect at very high frequencies, beyond the range considered in the present study.

The above equations therefore clearly illustrate that under both dark and TrVOR suppression conditions, $\mathrm{EM}_{\mathrm{C}}$ and $\mathrm{EM}_{\mathrm{I}}$ cells are predicted to exhibit different dynamic characteristics relative to the linear head acceleration signal $\ddot{H}_{\text {lin }}$.

\section{REFERENCES}

Angelaki DE (1991) Dynamic polarization vector of spatially tuned neurons. IEEE Trans Biomed Eng 38:1053-1060.

Angelaki DE (1998) Three-dimensional organization of otolith-ocular reflexes in rhesus monkeys. III. Responses to translation. J Neurophysiol 80:680-695.

Angelaki DE, Dickman JD (2000) Spatiotemporal processing of linear acceleration: primary afferent and central vestibular neuron responses. J Neurophysiol 84:2113-2132.

Angelaki DE, Hess BJM (2001) Direction of heading and vestibular control of binocular eye movements. Vision Res, in press.

Angelaki DE, McHenry MQ (1999) Short-latency primate vestibuloocular responses during translation. J Neurophysiol 82:1651-1654.

Angelaki DE, Bush GA, Perachio AA (1992) A model for the characterization of the spatial properties in vestibular neurons. Biol Cybern 66:231-240.

Angelaki DE, McHenry MQ, Hess BJM (2000a) Primate translational vestibulo-ocular reflexes. I. High frequency dynamics and threedimensional properties during lateral motion. J Neurophysiol 83:1637-1647.

Angelaki DE, McHenry MQ, Dickman JD, Perachio AA (2000b) Primate translational vestibulo-ocular reflexes. III. Effect of bilateral labyrinthine electrical stimulation. J Neurophysiol 83:1662-1676.

Angelaki DE, Newlands SD, Dickman JD (2000c) Primate translational vestibulo-ocular reflexes. IV. Changes after unilateral labyrinthectomy. J Neurophysiol 83:3005-3018.

Baker R, Berthoz A (1975) Is the prepositus hypoglossi nucleus the source of another vestibulo-ocular pathway? Brain Res 86:121-127.

Baker RG, Mano N, Shimazu H (1969) Postsynaptic potentials in abducens motoneurons induced by vestibular stimulation. Brain Res 15:577-580.

Barnes GR (1993) Visual-vestibular interaction in the control of head and eye movement: the role of visual feedback and predictive mechanisms. Prog Neurobiol 41:435-472.

Cannon SC, Robinson DA (1987) Loss of the neural integrator of the oculomotor system from brain stem lesions in monkey. J Neurophysiol 57:1383-1409.

Chen-Huang C, McCrea RA (1999) Effects of viewing distance on the responses of vestibular neurons to combined angular and linear vestibular stimulation. J Neurophysiol 81:2538-2557.

Cheron G, Godaux E (1987) Disabling of the oculomotor integrator by kainic acid injections in the prepositus-vestibular complex of the cat. J Physiol (Lond) 394:267-290.

Cullen KE, McCrea RA (1993) Firing behaviour of brain stem neurons during voluntary cancellation of the horizontal vestibuloocular reflex. I. Secondary vestibular neurons. J Neurophysiol 70:828-843.

Escudero M, Delgado-Garcia JM (1988) Behavior of reticular, vestibular and prepositus neurons terminating in the abducens nucleus of the alert cat. Exp Brain Res 71:218-222.

Escudero M, de la Cruz RR, Delgado-Garcia JM (1992) A physiological study of vestibular and prepositus hypoglossi neurones projecting to the abducens nucleus in the alert cat. J Physiol (Lond) 458:539-560.

Fernández C, Goldberg JM (1971) Physiology of peripheral neurons innervating semicircular canals of the squirrel monkey. II. Response to sinusoidal stimulation and dynamics of peripheral vestibular system. J Neurophysiol 34:661-675.

Fernández C, Goldberg JM (1976a) Physiology of peripheral neurons innervating otolith organs of the squirrel monkey. I. Response to static tilts and to long duration centrif ugal force. J Neurophysiol 39:970-984.

Fernández C, Goldberg JM (1976b) Physiology of peripheral neurons innervating otolith organs of the squirrel monkey. II. Directional selectivity and force-response relations. J Neurophysiol 39:985-995. 
Fernández C, Goldberg JM (1976c) Physiology of peripheral neurons innervating otolith organs of the squirrel monkey. III. Response dynamics. J Neurophysiol 39:996-1008.

Fuchs AF, Luschei ES (1970) Firing patterns of abducens neurons of alert monkeys in relationship to horizontal eye movement. J Neurophysiol 33:382-392.

Fuchs AF, Scudder CA, Kaneko CRS (1988) Discharge patterns and recruitment order of identified motoneurons and internuclear neurons in the monkey abducens nucleus. J Neurophysiol 60:1874-1895.

Galiana HL, Outerbridge JS (1984) A bilateral model for central neural pathways in the vestibulo-ocular reflex. J Neurophysiol 51:210-241.

Green AM (2000) Visual-vestibular interactions in a bilateral model of the rotational and translational vestibulo-ocular reflexes: an investigation of viewing-context-dependent reflex performance. $\mathrm{PhD}$ thesis, McGill University, Montreal, Canada.

Green AM, Galiana HL (1998) A hypothesis for shared central processing of canal and otolith signals. J Neurophysiol 80:2222-2228.

Green AM, Galiana HL (1999) An alternative approach to the central processing of canal and otolith signals. Ann NY Acad Sci 871:402-405.

Hikosaka O, Igusa Y, Imai H (1978) Firing pattern of prepositus hypoglossi and adjacent reticular neurons related to vestibular nystagmus in the cat. Brain Res 144:395-403.

Imagawa M, Isu N, Sasaki M, Endo K, Ikegami H, Uchino Y (1995) Axonal projections of utricular afferents to the vestibular nuclei and the abducens nucleus in cats. Neurosci Lett 186:87-90.

Kaneko CRS (1997) Eye movement deficits after ibotenic acid lesions of the nucleus prepositus hypoglossi in monkeys. I. Saccades and fixation. J Neurophysiol 78:1753-1768.

Keller EL, Kamath BY (1975) Characteristics of head rotation and eye movement-related neurons in alert monkey vestibular nucleus. Brain Res 100:182-187.

Langer T, Kaneko CRS, Scudder CA, Fuchs AF (1986) Afferents to the abducens nucleus in the monkey and cat. J Comp Neurol 245:379-400.

Lisberger SG, Pavelko TA (1988) Brain stem neurons in modified pathways for motor learning in the primate vestibulo-ocular reflex. Science 242:771-773.

Lisberger SG, Morris EJ, Tychsen L (1987) Visual motion processing and sensory-motor integration for smooth pursuit eye movements. Annu Rev Neurosci 10:97-129.

Lisberger SG, Pavelko TA, Broussard DM (1994) Responses during eye movements of brain stem neurons that receive monosynaptic inhibition from the flocculus and ventral paraflocculus in monkeys. J Neurophysiol 72:909-927.

McConville KMV, Tomlinson RD, Na E-Q (1996) Behavior of eye movement-related cells in the vestibular nuclei during combined rotational and translational stimuli. J Neurophysiol 76:3136-3148.

McCrea RA, Baker R (1985) Anatomical connections of the nucleus prepositus of the cat. J Comp Neurol 237:377-407.

McCrea RA, Yoshida K, Evinger C, Berthoz A (1981) The location, axonal arborization and termination sites of eye-movement-related secondary vestibular neurons demonstrated by intra-axonal HRP injection in the alert cat. In: Progress in oculomotor research (Fuchs A, Becker W, eds), pp 379-386. Amsterdam: Elsevier.

McCrea RA, Strassman A, May E, Highstein SM (1987) Anatomical and physiological characteristics of vestibular neurons mediating the horizontal vestibulo-ocular reflex of the squirrel monkey. J Comp Neurol 264:547-570.

McFarland JL, Fuchs AF (1992) Discharge patterns in nucleus prepositus hypoglossi and adjacent medial vestibular nucleus during horizontal eye movement in behaving macaques. J Neurophysiol 68:319-330.
McHenry MQ, Angelaki DE (2000) Primate translational vestibuloocular reflexes. II. Vergence and version responses during fore-aft motion. J Neurophysiol 83:1648-1661.

Musallam WS, Tomlinson RD (1999) Model for the translational vestibuloocular reflex (VOR). J Neurophysiol 82:2010-2014.

Paige GD, Tomko DL (1991a) Eye movement responses to linear head motion in the squirrel monkey. I. Basic characteristics. J Neurophysiol 65:1170-1182.

Paige GD, Tomko DL (1991b) Eye movement responses to linear head motion in the squirrel monkey. II. Visual-vestibular interactions and kinematic considerations. J Neurophysiol 65:1183-1196.

Precht W, Richter A, Grippo J (1969) Responses of neurones in cat's abducens nuclei to horizontal angular acceleration. Pflügers Arch 309:285-309.

Raphan T, Wearne S, Cohen B (1996) Modeling the organization of the linear and angular vestibulo-ocular reflexes. Ann NY Acad Sci 781:348-363.

Richter A, Precht W (1968) Inhibition of abducens motoneurones by vestibular nerve stimulation. Brain Res 11:701-705.

Robinson DA (1974) The effect of cerebellectomy on the cat's vestibuloocular integrator. Brain Res 71:195-207.

Robinson DA (1981) The use of control systems analysis in the neurophysiology of eye movements. Annu Rev Neurosci 4:463-503.

Schwarz U, Miles FA (1991) Ocular responses to translation and their dependence on viewing distance. I. Motion of the observer. J Neurophysiol 66:851-864.

Schwarz U, Busettini C, Miles FA (1989) Ocular responses to translation are inversely proportional to viewing distance. Science 245:1394-1396.

Schwindt PC, Richter A, Precht W (1973) Short latency utricular and canal input to ipsilateral abducens motoneurons. Brain Res 60:259-262.

Scudder CA, Fuchs AF (1992) Physiological and behavioral identification of vestibular nucleus neurons mediating the horizontal vestibuloocular reflex in trained rhesus monkeys. J Neurophysiol 68:244-264.

Skavenski AA, Robinson DA (1973) Role of abducens neurons in the vestibuloocular reflex. J Neurophysiol 36:724-738.

Snyder LH, King WM (1996) Behavior and physiology of the macaque vestibulo-ocular reflex response to sudden off-axis rotation: computing eye translation. Brain Res Bull 40:293-302.

Spencer RF, Wenthold RJ, Baker R (1989) Evidence for glycine as an inhibitory neurotransmitter of vestibular, reticular and prepositus hypoglossi neurons that project to the cat abducens nucleus. J Neurosci 9:2718-2736.

Telford L, Seidman SH, Paige GD (1997) Dynamics of squirrel monkey linear vestibuloocular reflex and interactions with fixation distance. J Neurophysiol 78:1775-1790.

Tomlinson RD, Robinson DA (1984) Signals in vestibular nucleus mediating vertical eye movements in the monkey. J Neurophysiol 51:1121-1135.

Uchino Y, Ikegami H, Sasaki M, Endo K, Imagawa M, Isu N (1994) Monosynaptic and disynaptic connections in the utriculo-ocular reflex arc of the cat. J Neurophysiol 71:950-958.

Uchino Y, Sasaki M, Sato H, Imagawa M, Suwa H, Isu N (1996) Utriculoocular reflex arc of the cat. J Neurophysiol 76:1896-1903.

Uchino Y, Sasaki M, Sato H, Imagawa M, Suwa H, Isu N (1997) Utricular input to cat extraocular motoneurons. Acta Otolaryngol Suppl 528:44-48.

Zhang Y, Partsalis AM, Highstein SM (1995) Properties of superior vestibular nucleus flocculus target neurons in the squirrel monkey. I. General properties in comparison with flocculus projecting neurons.J Neurophysiol 73:2261-2278, 